\title{
Artemisia annua L.: Essential oil and acetone extract composition and antioxidant capacity
}

\author{
Sandra C. Gouveia*, Paula C. Castilho \\ Centro de Química da Madeira, CCCEE, Universidade da Madeira, Campus Universitário da Penteada, piso 0, 9000 -390 Funchal, Portugal
}

\section{A R T I C L E I N F O}

\section{Article history:}

Received 26 May 2012

Received in revised form

21 September 2012

Accepted 17 December 2012

\section{Keywords:}

Artemisia annua

Caffeoylquinic acids

Phenolic

Essential oil

Antioxidant

\begin{abstract}
A B S T R A C T
Aerial parts of Artemisia annua growth in three different locations of Madeira Archipelago were studied. The essential oil composition was established by GC-MS and the main components were mono- and sesquiterpenes; artemisia ketone was not detected.

The presence of phenolic compounds in the acetone extracts was investigated by HPLC-DAD-ESI/MS ${ }^{n}$ and a diversified phenolic profile of 40 hydrocinnamic acid derivatives and glycosylated flavonoids was found. A few compounds were reported for the first time in Artemisia annua.

The antioxidant capacity of essential oils and extracts were measured by three different in vitro assays. For the essential oils, a very good antioxidant response was found and the extracts also showed a good antioxidant capacity, in particular as antiradical scavengers.
\end{abstract}

(c) 2012 Elsevier B.V. All rights reserved.

\section{Introduction}

The genus Artemisia (family of Asteraceae) includes more than 300 species, mainly small herbs and shrubs (Yoon et al., 2011).

There are several reports (Carvalho et al., 2011) describing Artemisia plants as dietary foods and as traditional herbal medicines against inflammatory diseases, infections by fungi, bacteria and viruses, gastric ulcer, cancer among others.

One of the most studied Artemisia plants is Artemisia annua L., commonly known as "qinghao" or "annual wormwood". It is a plant used for many centuries in Chinese folk medicine for the treatment of malaria and fever and several bioactive metabolites have been reported, the most important of them being artemisinin, an endoperoxide sesquiterpene lactone (Castilho et al., 2008).

Artemisia annua leaves have a high content of essential oil (EO) containing cineole, $\alpha$-pinene, camphene, borneol, camphor, germacrene-D and artemisia ketone (Bora and Sharma, 2011).

The essential oil of Artemisia annua is referenced as having antifungal and antimicrobial activity (Juteau et al., 2002; Verma et al., 2011). In addition to these, other biological properties are associated to more polar extracts of Artemisia annua such

\footnotetext{
* Corresponding author at: Kemihuset, Linnaeus väg 10, KB6A14, Umeå universitet, SE-901 87 Umeå, Sweden. Tel.: +46 907865182.

E-mail address: sandra.gouveia@chem.umu.se (S.C. Gouveia).
}

as antimalarial, antibacterial, anti-inflammatory, antitumor and antiulcerogenic (Bhakuni et al., 2001; Ćavar et al., 2012). The antioxidant capacity referred in the literature is associated to the high flavonoid content and diversified types of compounds (Brisibe et al., 2009; Ferreira et al., 2010). Also, in vitro antimalarial and anticancer activity of artemisinin and its derivatives is enhanced by the presence certain flavonoids (Ferreira et al., 2010).

The phenolic composition of Artemisia annua has been described using HPLC-MS/MS techniques by (Lai et al., 2007), (Han et al., 2008) and (Carbonara et al., 2012); Carvalho and co-workers (Carvalho et al., 2011) reported the HPLC-DAD quantification of several phenolic compounds.

The main phenolic compounds reported were flavonoids and hydroxycinnamates. Based on literature survey, the chemical composition and biological properties of Artemisia annua can extensively differ according to their geographical origin and how the plant material is processed. The interest in the potential applications of this plant is still increasing and in the present study we analysed Artemisia annua plants grown in Madeira Archipelago, previously reported as having ca. $1 \%$ of artemisin (Castilho et al., 2008).

The main goals were to establish the phenolic composition of crude acetone and methanol extracts by high-performance liquid chromatography with diode array detection coupled with mass spectrometry (HPLC-DAD-ESI/MS ${ }^{\mathrm{n}}$ ); the EO composition by GCMS and the antioxidant capacity of the EO and the extracts were evaluated by three different assays (DPPH, ABTS and FRAP). 


\section{Materials and methods}

\subsection{Chemicals and standards}

The following reagents were purchased from Merck (Darmstadt, Germany): disodium phosphate dodecahydrated (99\%), potassium persulfate (99\%), ferrous sulfate heptahydrate (99\%), glacial acetic acid (100\%), sodium carbonate (p.a.) and sodium chloride (99.5\%). 2,2-diphenyl-1-picrylhydrazyl (DPPH) (>95\%), Trolox ( $\geq 99.8 \%$, HPLC), 2,2'azinobis-(3-ethylbenzthiazoline-6-sulfonic acid)(ABTS) ( $\geq 99 \%$, HPLC), 2,4,6-Tri(2-Pyridyl)-s-triazine (TPTZ) ( $\geq 99.0 \%$, TLC) and Folin-Ciocalteu's phenol reagent were purchased from Fluka (Lisbon, Portugal). Potassium chloride ( $>99.5 \%)$, gallic acid $(99 \%$, HPLC), potassium acetate (p.a.), rutin ( $\geq 98 \%$, HPLC) and ferric chloride hexahydrate (97-100\%) were purchased from Panreac (Barcelona, Spain); potassium dihydrogen phosphate (99.5\%), aluminium chloride (98\%) and sodium acetate trihydrate (pure) were purchased from Riedel-de Haën (Hanover, Germany).

All solvents used for plant extraction were AR grade, purchased from Fisher (Lisbon, Portugal). HPLC-MS grade acetonitrile (99.9\%, LabScan, Gliwice, Poland) and ultra-pure water (Milli-Q Waters purification system, EUA) were used for HPLC analysis.

Standards prepared in ethanol $(100 \mu \mathrm{g} / \mathrm{mL})$ : apigenin $(>99 \%)$, luteolin ( $>99 \%)$, quercetin ( $>99 \%), p$-coumaric acid (>99\%) and caffeic acid (>99\%) from Extrasynthese (Lyon, France), kaempferol (>99\%) and 5-O-caffeoylquinic acid (99\%) from Acros Organics (Geel, Belgium). 1,3-O-dicaffeoylquinic acid, 1,5-O-dicaffeoylquinic acid, 3,4-O-dicaffeoylquinic acid, 3,5-O-dicaffeoylquinic acid, 4,5$O$-dicaffeoylquinic acid and 3,4,5-O-tricaffeoylquinic acid ( $>98 \%$ by HPLC for all) were obtained from Chengdo Biopurify Phytochemicals, Ltd China (Sichuan, China).

\subsection{Plant material and sample preparation}

Samples of Artemisia annua were obtained from seeds of artemisinin rich hybrids (CPQBA $\times$ POP) kindly offered by UNICAMP, Brazil and cultivated in three different locations of Madeira Archipelago (Preces, Jardim Botânico and Porto Santo).

Before flowering, the whole plants were cut and dried. Plants grown in Madeira Botanical Garden (Jardim Botânico) experimental field reached about $3.5 \mathrm{~m}$ high. These plants were divided into two lots and one of them was dried under direct sunlight in order to maximize artemisinin production (Castilho et al., 2008). Leaves from the top third of plants were processed separately from those of the rest of the plant. Vouchers were deposited in the Madeira Botanical Garden Herbarium collection.

The dried plant material was ground to fine powder in a mechanic grinder. Portions of this powdered plant were separately extracted by solid liquid extraction, using two solvents (acetone and methanol): The plant material $(50 \mathrm{~g}$ ) was macerated with $500 \mathrm{~mL}$ of solvent, at room temperature, for $24 \mathrm{~h}$.

In all cases, the solutions were filtered and concentrated under reduced pressure in a rotary evaporator $\left(40^{\circ} \mathrm{C}\right)$ and kept in the dark at $-20^{\circ} \mathrm{C}$ until tested.

Essential oils were obtained by hydrodistillation in a Clevenger type apparatus.

\subsection{Essential oil analysis by GC-MS analysis}

Qualitative and quantitative composition of essential oils was performed by GC and GC-MS analysis, using a HP 5889 series II and a Varian Saturn 3 ion trap system, respectively in both cases, columns DB-5 (30 m $\times 0.15 \mathrm{~mm} \times 0.25 \mu \mathrm{m}$; J. W. Scientific) were used; helium N60 was used as carrier gas. The analysis conditions were: initial temperature $40^{\circ} \mathrm{C}$ for $14 \mathrm{~min}$, gradient of $1.5^{\circ} \mathrm{C} / \mathrm{min}$ up to $250^{\circ} \mathrm{C}$, isotherm for $10 \mathrm{~min}$. Injector (and transfer line) temperature was $270^{\circ} \mathrm{C} .1 \mu \mathrm{L}$ was injected in splitless mode. GC-MS analysis was performed by ESI in a mass range 24 to 400 $\mathrm{m} / \mathrm{z}$ with a delay time of $2 \mathrm{~min}$.

Identification of components was performed by comparing the mass spectra of those of the available NIST database (GC-MS) and by their relative retention index (Kovacs Index, KI) towards a mixture of alkanes $C_{4}$ to $C_{26}$, injected in the same experimental conditions (GC-FID).

\subsection{HPLC-DAD-ESI/MS ${ }^{n}$ analysis}

\subsubsection{Liquid chromatography}

Stock solutions with concentrations $(\mathrm{m} / \mathrm{v})$ of $5 \mathrm{mg} / \mathrm{mL}$ were prepared by dissolving each dried extract in initial HPLC mobile phase (Acetonitrile/Water (20/80, v/v)). These solutions were filtered through $0.45 \mathrm{~mm}$ Nylon micropore membranes prior to use and $10 \mu \mathrm{L}$ were injected for HPLC-DAD-ESI/MS ${ }^{n}$ analysis. Three independent assays were performed for each sample.

The HPLC separation was carried out on a Dionex ultimate 3000 series instrument (California, EUA) coupled to a binary pump, a diode-array detector (DAD), an autosampler and a column compartment.

Samples were separated on a Phenomenex Gemini $C_{18}$ column ( $5 \mu \mathrm{m}, 250 \mathrm{~mm} \times 3.0 \mathrm{~mm}$ i.d.; Phenomenex) with a sample injection volume of $10 \mu \mathrm{L}$. The mobile phase was composed of acetonitrile (A) and water/formic acid (100/0.1, v/v) (B). A gradient program was used as follows: $20 \% \mathrm{~A}$ ( $0 \mathrm{~min}), 25 \% \mathrm{~A}$ (10 min), $25 \% \mathrm{~A}$ (20 min), 50\% A (40 min), 100\% A (42-47 min), 20\% A (49-55 min). The mobile phase flow rate was $0.4 \mathrm{~mL} / \mathrm{min}$; the chromatogram was recorded at $280 \mathrm{~nm}$ and $350 \mathrm{~nm}$ and spectral data for all peaks were accumulated in the range of $190-400 \mathrm{~nm}$. Column temperature was controlled at $30^{\circ} \mathrm{C}$.

\subsubsection{Mass spectrometry}

For HPLC-ESI/MS ${ }^{\mathrm{n}}$ analysis, a model 6000 ion trap mass spectrometer (Bruker Esquire, Bremen, Germany) fitted with an ESI source operating in the negative mode was used. Data acquisition and processing were performed using Esquire control software. Negative ion mass spectra of the column eluate were recorded in the range $m / z 100-1000$ at a scan speed of $13,000 \mathrm{Da} / \mathrm{s}$. High purity nitrogen $\left(\mathrm{N}_{2}\right)$ was used both as drying gas at a flow of $10.0 \mathrm{~mL} / \mathrm{min}$ and as a nebulizing gas at a pressure of 50 psi. The nebulizer temperature was set at $365^{\circ} \mathrm{C}$ and a potential of $+400 \mathrm{~V}$ was used on the capillary. Ultra-high-purity helium $(\mathrm{He})$ was used as collision gas at a pressure of $1 \times 10^{-5}$ mbar and the collision energy was set at $40 \mathrm{~V}$.

The acquisition of MS ${ }^{\mathrm{n}}$ data was made in auto $\mathrm{MS}^{\mathrm{n}}$ mode, with an isolation width of $4.0 \mathrm{~m} / z$. For MS ${ }^{\mathrm{n}}$ analysis, the mass spectrometer was scanned from 10 to $1000 \mathrm{~m} / z$ with fragmentation amplitude of $1.0 \mathrm{~V}\left(\mathrm{MS}^{\mathrm{n}}\right.$ up to $\left.\mathrm{MS}^{4}\right)$ and two precursor ions.

\subsection{Total phenolic content (TPC)}

The total phenolic content the extracts was determined following the Folin-Ciocalteu method (Zheng and Wang, 2001) with some modifications (Gouveia and Castilho). Each sample aliquot of $50 \mu \mathrm{L}$ $(10 \mathrm{mg} / \mathrm{mL}, \mathrm{w} / \mathrm{v})$ was mixed with $1.25 \mathrm{~mL}$ of Folin-Ciocalteu reagent (diluted $1: 10$ fold) and $1 \mathrm{~mL}$ of $7.5 \%$ sodium carbonate solution. The mixture was incubated for $30 \mathrm{~min}$ at room temperature and then absorbance was measured at $\lambda=765 \mathrm{~nm}$. The final results were expressed as milligrams of gallic acid equivalents per $100 \mathrm{~g}$ of dried plant (mg GAE/100 g). 


\subsection{DPPH radical scavenging activity}

For the DPPH assay, $100 \mu \mathrm{L}$ of the sample solutions $(10 \mathrm{mg} / \mathrm{mL})$ were added to $3.5 \mathrm{~mL}$ of a $0.06 \mathrm{mM}$ methanol DPPH radical solution (Gouveia and Castilho, 2012a, b). The decrease in absorbance at $\lambda=516 \mathrm{~nm}$ was measured during $30 \mathrm{~min}$. The DPPH radical scavenging effect of the extracts was expressed as $\mu$ mol Trolox equivalent per 100 gram of dried plant ( $\mu$ mol eq. Trolox/100 g dried plant) for plant extracts and as $\mu \mathrm{mol}$ Trolox equivalent per $\mathrm{mL}$ of essential oil ( $\mu \mathrm{mol}$ eq. Trolox/mL EO) for essential oils.

\subsection{ABTS $^{\bullet+}$ radical scavenging activity}

The $\mathrm{ABTS}^{\bullet+}$ radical working solution was prepared by mixing $50 \mathrm{~mL}$ of $2 \mathrm{mM} \mathrm{ABTS}^{\bullet+}$ solution with $200 \mu \mathrm{L}$ of $70 \mathrm{mM}$ potassium persulfate solution (Gouveia and Castilho, 2012a,b). This mixture was kept in the dark for $16 \mathrm{~h}$ at room temperature. For each analysis, the $\mathrm{ABTS}^{\bullet+}$ solution was diluted with $\mathrm{pH} 7.4$ phosphate buffered saline (PBS) solution to an initial absorbance of $0.700 \pm 0.021$ at $734 \mathrm{~nm}$. This solution was freshly prepared for each analysis. For the assessment of the radical scavenging activity, an aliquot of $100 \mu \mathrm{L}$ $\left(10 \mathrm{mg} / \mathrm{mL}, \mathrm{w} / \mathrm{v}\right.$ ) was added to $1.8 \mathrm{~mL}$ of $\mathrm{ABTS}^{\bullet+}$ working solution and the absorbance decrease, at a $\lambda=734 \mathrm{~nm}$, was recorded during $6 \mathrm{~min}$. Results were expressed as $\mu \mathrm{mol}$ Trolox equivalent per $100 \mathrm{~g}$ of dried plant ( $\mu \mathrm{mol}$ eq. Trolox/100 g dried plant) for plant extracts and as $\mu \mathrm{mol}$ Trolox equivalent per $\mathrm{mL}$ of essential oil ( $\mu \mathrm{mol}$ eq. Trolox/mL EO) for essential oils.

\subsection{Ferric reducing activity (FRAP assay)}

The ferric reducing ability of the extracts was measured based on the FRAP assay (Benzie and Strain, 1996). FRAP reagent was prepared daily by mixing $2.5 \mathrm{~mL}$ of solution ferric trichloride hexahydrate $(20 \mathrm{mM}), 2.5 \mathrm{~mL}$ of solution TPTZ $(10 \mathrm{mM}$ in $40 \mathrm{mM}$ of hydrochloric acid) and $25 \mathrm{~mL}$ of acetate buffer $0.3 \mathrm{M}(\mathrm{pH} 3.6)$ and incubating at $37^{\circ} \mathrm{C}$. For each analysis, $30 \mu \mathrm{L}$ of methanolic solution $(1 \mathrm{mg} / \mathrm{mL}, \mathrm{w} / \mathrm{v})$ were added to $180 \mu \mathrm{L}$ of distilled water and $1.8 \mathrm{~mL}$ of FRAP solution. The increase of absorbance was recorded at $\lambda=593 \mathrm{~nm}$ in $15 \mathrm{~s}$ intervals, during $30 \mathrm{~min}$ at $37^{\circ} \mathrm{C}$. The FRAP results were expressed as mmol Iron(II) sulfate heptahydrate per $\mathrm{mg}$ of dried plant ( $\mathrm{mmol} \mathrm{Fe}(\mathrm{II}) / \mathrm{mg}$ ) for plant extracts and as mmol Iron(II) sulfate heptahydrate per $\mathrm{mL}$ of essential oil ( $\mathrm{mmol} \mathrm{Fe}(\mathrm{II}) / \mathrm{mL}$ EO) for essential oils.

\section{Results and discussion}

\subsection{Essential oil composition}

The chemical composition of essential oils obtained from dried leaves is presented in Table 1.

Similar results have been published by Magalhães et al. (2004), who produced this hybrid and cultivated it in Brazil. Comparing these sets of results it was clear that the composition of essential oil was not substantially affected by climate, location or cultivating condition but it is intrinsic to the plant. Even drying in direct sunshine or shade did not affect much the essential oil composition, with a little loss of the more volatile components being observed for the plant dried under sunlight. The $C P Q B A \times P O P$ hybrid has no thujone derivatives or artemisia ketone, harmful compounds normally present in wild-type Artemisia annua L. (Ćavar et al., 2012; Reale et al., 2011). The only possible troublesome, in terms of safety, component could be camphor (ca. 40\%). The pair 1,8-cineole/camphor as the two major components in essential oils has been found not only in Artemisia annua (Ćavar et al., 2012; Holm et al., 1997; M R, 2009) but in several other Artemisia subspecies (Kordali et al., 2005; Lopes-Lutz et al., 2008; Shang et al., 2012).

\subsection{Phenolic composition by HPLC-DAD-ESI/MS ${ }^{n}$}

Comparison between the HPLC-DAD-ESI/MS ${ }^{n}$ screening of methanolic and acetone extracts showed that, for the methanolic extract, the number of peaks detected was lower and with reduced chromatographic resolution leading to a poor fragmentation of the detected compounds. This can be associated to matrix effects and high molecular weight compounds common in more polar solvents extractions. Several authors (Kallithraka et al., 1995; Korekar et al., 2011) have evaluated methanol versus acetone as extraction solvents for phenolic compounds with clear advantage for acetone. Our choice for this solvent resulted from our previous work where it was the best to extract artemisinin. The acetone extracts from plants cultivated in three different locations were very similar between them, thus we only present the chromatogram and fragmentation data for one of them (Preces).

The HPLC-DAD-ESI/MS ${ }^{n}$ base peak chromatogram profile for acetone extract is shown in Fig. 1.

The identification and characterization of the detected compounds was made by comparison of the HPLC retention, UV and mass spectra with those of standard compounds. Since only a limited number of standard compounds was available, structures of unknown compounds were established largely based on their tandem MS ${ }^{\mathrm{n}}$ fragmentation behaviour.

Among the detected compounds, we found typical hydroxycinnamic acid UV absorptions ( $\lambda \max 230-240,300$ sh. $320-340 \mathrm{~nm}$ ) and flavonoids characteristic UV absorptions: flavonols derivatives exhibited two maximum absorptions at $250-270 \mathrm{~nm}$ and $320-360 \mathrm{~nm}$, derived from the aglycone A and B rings, respectively. Peaks corresponding to flavones conjugates showed three absorptions at $210-230 \mathrm{~nm}, 250-280 \mathrm{~nm}$ and $330-350 \mathrm{~nm}$ (Mabry et al., 1970).

In our study, 38 compounds were tentatively identified and some of them are reported for the first time for Artemisia annua.

Fig. 2 represents the chemical structures of main classes of compounds detected in Artemisia annua. Table 2 shows the experimental analytical data: retention time $\left(t_{R}\right)$, wavelength of maximum absorbance $\left(\lambda_{\max }\right)$, deprotonated molecular ions $[\mathrm{M}-\mathrm{H}]^{-}$, and most important $\mathrm{MS}^{\mathrm{n}}$ fragment ions for each peak.

Usually, the base peak in the $\mathrm{MS}^{1}$ spectrum was assigned as the deprotonated molecular ion $[\mathrm{M}-\mathrm{H}]^{-}$. When isomers were observed, their identification was performed based on literature data.

\subsubsection{Identification of flavonoids}

The occurrence of flavonoids, in particular those conjugated with one or more sugar moieties, in Artemisia species has been previously reported (Ferreira et al., 2010, Gouveia and Castilho, Han et al., 2008; Lai et al., 2007). In HPLC-ESI/MS ${ }^{n}$ screening of Artemisia annua, mainly apigenin and quercetin conjugates have been reported. In the present work, in addition to these two types of aglycones, luteolin, isorhamnetin and kaempferol derivatives compounds were also identified.

The tandem MS/MS experiments of the flavonoids resulted on the deprotonated molecular ion $\left([\mathrm{M}-\mathrm{H}]^{-}\right)$and the deprotonated aglycone ion $\left(\mathrm{Y}_{0}^{-}\right)$as a result of the loss of the sugar unit.

Most of the detected flavonoids were in their glycosylated form and/or esterified with acyl groups. But two aglycones were detected on its free form - luteolin and apigenin.

3.2.1.1. Free aglycones ( 36 and $\mathbf{4 0})$. Compound $\mathbf{3 6}\left(t_{R}=27.7 \mathrm{~min}\right)$ was identified as luteolin by comparison with a reference standard (data not shown). In the $\mathrm{MS}^{\mathrm{n}}$ experiments the $[\mathrm{M}-\mathrm{H}]^{-}$ion at $\mathrm{m} / \mathrm{z}$ 285 gave several typical luteolin fragment ions at $m / z$ 199, 217 ([M$\left.\left.\mathrm{H}-\mathrm{C}_{3} \mathrm{O}_{2}\right]^{-}\right), 175\left(\left[\mathrm{M}-\mathrm{H}-\mathrm{C}_{3} \mathrm{O}_{2}-\mathrm{C}_{2} \mathrm{H}_{2} \mathrm{O}\right]^{-}\right)$and $241\left(\left[\mathrm{M}-\mathrm{H}-\mathrm{CO}_{2}\right]^{-}\right)$.

Compound $40\left(t_{\mathrm{R}}=33.9 \mathrm{~min}\right)$ exhibited a $[\mathrm{M}-\mathrm{H}]^{-}$ion at $\mathrm{m} / z 269$ and the its $\mathrm{MS}^{\mathrm{n}}$ fragmentation presented main fragment ions at 
Table 1

Composition of the essential oil (EO) from leaves of Artemisia annuagrowth in Preces, Porto Santo and JardimBotânico (dried under direct sunlight and in the shade).

\begin{tabular}{|c|c|c|c|c|c|c|}
\hline KI ref ${ }^{*}$ & $\mathrm{KI}$ & Compound & Preces & Porto Santo & JardimBotânico Shade & JardimBotânico Sunshine \\
\hline 937 & 937 & $\alpha$-pinene & 3.58 & 1.55 & 3.19 & 2.79 \\
\hline 953 & 954 & camphene & 0.28 & 0.01 & 0.23 & 0.31 \\
\hline 990 & 902 & $\beta$-pinene & 0.60 & 0.27 & 1.35 & 0.88 \\
\hline 1018 & 1017.0 & $\alpha$-terpinene & 0.68 & 0.68 & 0.77 & 0.78 \\
\hline 1026 & 1027.0 & p-cymene & 3.32 & 1.23 & 2.10 & 3.31 \\
\hline 1030 & 1031.0 & limonene & 0.23 & 0.05 & 0.39 & 0.24 \\
\hline 1030 & 1035.0 & 1,8-cineole & 11.6 & 6.54 & 9.87 & 7.92 \\
\hline 1059 & 1061.0 & $\gamma$-terpinene & 1.27 & 1.22 & 1.35 & 1.28 \\
\hline 1141 & 1141.0 & trans-pinocarveol & 0.51 & 0.88 & 0.86 & 0.64 \\
\hline 1143 & 1149.0 & camphor & 42.8 & 45.5 & 42.0 & 44.33 \\
\hline 1165 & 1173.0 & borneol & 2.50 & 8.96 & 2.58 & 4.74 \\
\hline 1177 & 1180.0 & terpinen-4-ol & 2.80 & 2.59 & 2.45 & 2.51 \\
\hline 1194 & 1196.0 & myrtenol & 1.23 & 1.21 & 1.00 & 1.08 \\
\hline 1217 & 1222.0 & trans-carveol & 1.77 & 1.76 & 1.79 & 1.55 \\
\hline 1242 & 1247.0 & l-carvone & 1.03 & 0.52 & 0.30 & 0.78 \\
\hline 1345 & 1346.0 & $\alpha$-cubebene & 0.10 & 0.10 & 0.08 & 0.13 \\
\hline 1351 & 1353.0 & eugenol & 1.13 & 0.66 & 1.23 & 1.09 \\
\hline 1376 & 1373.0 & $\alpha$-copaene & 1.75 & 0.17 & 0.82 & 1.15 \\
\hline 1389 & 1388.0 & $\beta$-cubebene & 1.93 & 1.31 & 1.02 & 1.66 \\
\hline 1394 & 1392.0 & cis-jasmone & 0.24 & 0.72 & 0.24 & 0.48 \\
\hline 1428 & 1417.0 & $\beta$-caryophyllene & 7.77 & 10.21 & 6.44 & 8.65 \\
\hline 1444 & 1452.0 & $\alpha$-humulene & 0.46 & 0.94 & 6.70 & 0.94 \\
\hline 1480 & 1479.0 & germacrene-D & 7.15 & 8.41 & 8.61 & 7.77 \\
\hline 1485 & 1486.0 & $\beta$-selinene & 1.93 & 3.09 & 2.78 & 3.04 \\
\hline 1494 & 1493.0 & bicyclogermacrene & 3.29 & 1.4 & 1.86 & 1.95 \\
\hline
\end{tabular}

* See http://www.pherobase.com/database/kovats/kovats-index.php.

$m / z 225\left(\left[\mathrm{M}-. \mathrm{H}-\mathrm{CO}_{2}\right]^{-}\right), 151\left(1,3 \mathrm{~A}^{-}\right)$and $149\left({ }^{1,4} \mathrm{~B}^{-}+2 \mathrm{H}\right)$. Comparing these results to those obtained for a standard solution of apigenin, $\mathbf{4 0}$ was identified as free apigenin.

Two types of flavonoid glycosilation were observed: $O$ glycosilation and $C$-glycosilation.

The identification of these two groups was based on the tandem $\mathrm{MS}^{\mathrm{n}}$ fragmentation experiments. For C-glycosylated compounds the sugar unit is linked to the aglycone with a carbon-carbon bond that is resistant to rupture.

The representative $\mathrm{MS}^{\mathrm{n}}$ fragments for $C$-glycosylated flavonoids are related to the sugar part and occurs at $m / z^{0,2} \mathrm{X}^{-}[\mathrm{M}-\mathrm{H}-120]^{-}, 0,3$ $\mathrm{X}^{-}$[M-H-90 $^{-}$and ${ }^{0,2} \mathrm{X}^{-}$[M-H-60 $]^{-}$(Cuyckens and Claeys, 2004). The non-observation of deprotonated aglycone ion, $\mathrm{Y}_{0}^{-}$, makes identification process harder.

For $O$-glycosylated compounds the sugar moieties are lost as neutral fragments and the flavonoid structure is more easily identified due to aglycone bonds cleavages.

3.2.1.2. C-glycosides $(\mathbf{7}, \mathbf{8}, \mathbf{9}, \mathbf{1 2}$ and 13). For these compounds UV spectra showed maximum absorptions bands at $\lambda=225,272$ and
$314 \mathrm{~nm}$ identical to characteristics absorptions bands of flavones (Mabry et al., 1970). However, the tandem MS $^{n}$ experiments revealed a different pattern to that known for $O$-glycosylated flavones.

Compound $7\left(t_{\mathrm{R}}=5.4 \mathrm{~min}\right)$ and $8\left(t_{\mathrm{R}}=5.8 \mathrm{~min}\right)$ showed a [M$\mathrm{H}]^{-}$ions at $m / z 563$. The $\mathrm{MS}^{2}$ fragmentation revealed a behaviour typical of the asymmetrical di-C-glycosides with fragment ions at $[\mathrm{M}-\mathrm{H}-210]^{-},[\mathrm{M}-\mathrm{H}-90]^{-}$and $[\mathrm{M}-\mathrm{H}-60]^{-}$. The neutral loss of $60 \mathrm{Da}$ $\left({ }^{0,3} \mathrm{X}^{-}\right)$indicates the presence of a pentose moiety.

Compound $\mathbf{8}$ has been previously described by our group in Artemisia argentea (Gouveia and Castilho, 2011a,b) and was characterized as apigenin-6-C-hexoside-8- $C$-pentoside.

Compound $\mathbf{7}$ similar to $\mathbf{8}$ had $\mathrm{MS}^{3}$ fragment ions at $\mathrm{m} / \mathrm{z}$ $353\left[{ }^{0,2} \mathrm{X}_{\mathrm{i}}{ }^{0,2} \mathrm{X}_{\mathrm{j}}\right]^{-}$and $383\left[^{0,2} \mathrm{X}_{\mathrm{i}}{ }^{0,3} \mathrm{X}_{\mathrm{j}}\right]^{-}$suggesting apigenin as the aglycone (Vukics et al., 2008). However, contrarily to compound 8, 7 did not present an intense $[\mathrm{M}-\mathrm{H}-60]^{-}$ion at $\mathrm{m} / \mathrm{z} 503$ (2.9\% of base peak). Based on these evidences and knowing that 6 - $C$-pentoside-8-C-hexoside elutes before the isomer 6 - $C$-hexoside- $8-C$-pentoside, compound 7 was identified as apigenin-6- $C$-pentoside-8- $C$-hexoside.

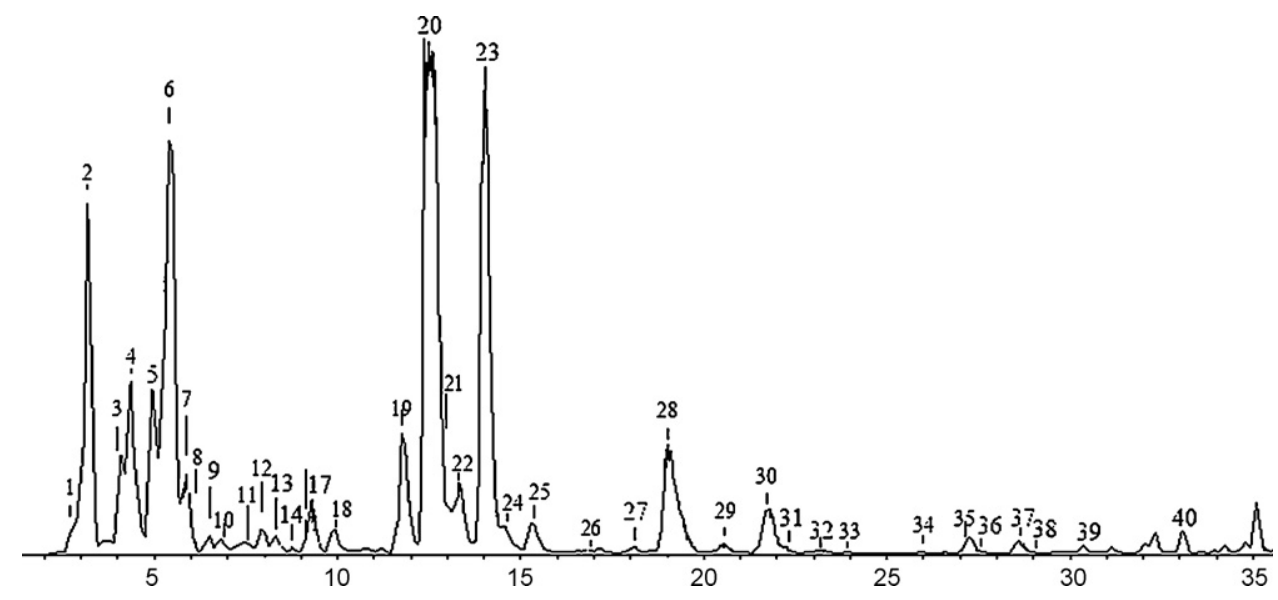

Fig. 1. HPLC-DAD-ESI/MS ${ }^{\mathrm{n}}$ analysis of the acetone extracts of Artemisia annua total aerial parts - HPLC-MS negative ion ESI/MS ${ }^{\mathrm{n}}$ base peak chromatogram (BPC). 
Table 2

Characterization of phenolic components of the acetone extract from Artemisia annua by HPLC-DAD-ESI/MS ${ }^{\mathrm{n}}$.

\begin{tabular}{|c|c|c|c|c|c|}
\hline No. & $t_{\mathrm{R}}(\min )$ & Identification & $\lambda_{\max }(\mathrm{nm})$ & {$[\mathrm{M}-\mathrm{H}]^{-}(m / z)$} & HPLC-DAD-ESI/MS ${ }^{n} m / z$ (\% base peak) \\
\hline 1 & 2.8 & $\begin{array}{l}\text { Caffeic acid hexoside } \\
\text { derivative }\end{array}$ & 275,304 & 473 & $\begin{array}{l}\operatorname{MS}^{2}[473]: 342(14.4), 341(100), 179(13.8), 161(13.8), 131(29.2) \\
\text { MS }^{3}[473 \rightarrow 341]: 179(100), 161(43.7), 143(10.8), 119(44.2), 101(11.3) \\
\text { MS }^{4}[473 \rightarrow 341 \rightarrow 179]: 119(92.0), 113(54.0), 101(63.2), 89(100)\end{array}$ \\
\hline 2 & 3.1 & Quinic acid & - & 191 & $\begin{array}{l}\text { MS }^{2}[191]: 173(74.9), 127(100), 111(47.0), 109(69.7), 85(70.7) \\
\text { MS }^{3}[191 \rightarrow 127]: 163(30.8), 109(69.7), 99(100)\end{array}$ \\
\hline 3 & 4.0 & $\begin{array}{l}\text { Kaempferol-3,7-di-O- } \\
\text { hexoside }\end{array}$ & - & 609 & $\begin{array}{l}\text { MS }^{2}[609]: 449(18.2), 448(11.6), 447(100), 285(34.9) \\
\text { MS }^{3}[609 \rightarrow 447]: 327(7.6), 284(73.0), 285(100), 255(62.3) \\
\text { MS }^{4}[609 \rightarrow 447 \rightarrow 284]: 256(31.1), 255(100), 151(34.2)\end{array}$ \\
\hline 4 & 4.4 & 3-O-Caffeoylquinic acid & $241,300,324$ & 353 & $\begin{array}{l}\mathrm{MS}^{2}[353]: 191(100), 179(51.4), 135(15.9) \\
\text { MS }^{3}[353 \rightarrow 191]: 173(100), 171(16.9), 127(56.8), 111(30.2), 109(39.2) \\
85(37.4) \\
\text { MS }^{4}[353 \rightarrow 191 \rightarrow 127]: 109(100), 99(90.9), 85(12.9)\end{array}$ \\
\hline $5^{*}$ & 5.0 & 5-O-Caffeoylquinic acid & $242,300,325$ & 353 & $\begin{array}{l}\operatorname{MS}^{2}[707]: 353(100) \\
\operatorname{MS}^{2}[353]: 191(100) \\
\operatorname{MS}^{3}[707 \rightarrow 353]: 191(100) \\
\text { MS }^{3}[353 \rightarrow 191]: 173(57.1), 127(100), 109(55.9), 85(97.7) \\
\text { MS }^{4}[707 \rightarrow 353 \rightarrow 191]: 173(100), 127(63.7), 135(21.8), 111(17.4) \\
\operatorname{MS}^{4}[353 \rightarrow 191 \rightarrow 127]: 109(100)\end{array}$ \\
\hline 6 & 5.2 & 4-O-Caffeoylquinic acid & $240,300,323$ & 353 & $\begin{array}{l}\mathrm{MS}^{2}[353]: 191(18.4), 179(58.3), 173(100), 135(8) \\
\operatorname{MS}^{3}[353 \rightarrow 173]: 111(30.8), 93(100)\end{array}$ \\
\hline 7 & 5.4 & $\begin{array}{l}\text { Apigenin-6-C- } \\
\text { pentoside-8-C- } \\
\text { hexoside. }\end{array}$ & $224,273,314$ & 563 & $\begin{array}{l}\mathrm{MS}^{2}[563]: 503(2.9), 473(98.0), 443(78.2), 383(83.5), 353(100) \\
\mathrm{MS}^{3}[563 \rightarrow 353]: 326(13.4), 325(100), 297(78.2) \\
\mathrm{MS}^{4}[563 \rightarrow 352 \rightarrow 325]: 298(5.1), 297(100)\end{array}$ \\
\hline 8 & 5.9 & $\begin{array}{l}\text { Apigenin-6- } C \text { - } \\
\text { hexoside- } 8-C- \\
\text { pentoside. }\end{array}$ & $224,273,314$ & 563 & $\begin{array}{l}\mathrm{MS}^{2}[563]: 503(59.3), 473(68.6), 443(100), 383(63.0), 353(63.2) \\
\mathrm{MS}^{3}[563 \rightarrow 443]: 383(50.0), 354(12.0), 353(100) \\
\mathrm{MS}^{4}[563 \rightarrow 443 \rightarrow 353]: 326(35.1), 325(100), 297(56.2)\end{array}$ \\
\hline 9 & 6.5 & $\begin{array}{l}\text { Apigenin- } 6-C- \\
\text { rhamnoside-8- } C \text { - } \\
\text { hexoside }\end{array}$ & $224,272,313$ & 577 & $\begin{array}{l}\mathrm{MS}^{2}[577]: 559(21.9), 503(2.8), 487(28.3), 473(40.0), 457(53.1), 353 \\
(100) \\
\mathrm{MS}^{3}[577 \rightarrow 353]: 326(18.3), 325(100), 297(71.2) \\
\mathrm{MS}^{4}[577 \rightarrow 353 \rightarrow 325]: 297(100)\end{array}$ \\
\hline 10 & 6.9 & 3-O-Feruloylquinic acid & 326 & 367 & $\begin{array}{l}\text { MS }^{2}[367]: 193(100), 191(1.5), 173(3.7) \\
\text { MS }^{3}[367 \rightarrow 193]: 149(31.9), 134(100), 109(10.3)\end{array}$ \\
\hline 11 & 7.4 & 5-O-Feruloylquinic acid & 327 & 367 & $\begin{array}{l}\operatorname{MS}^{2}[367]: 191(100) \\
\operatorname{MS}^{3}[367 \rightarrow 191]: 179(100), 134(53.0), 111 \text { (68.6), } 127 \text { (55.5), } 109(12.7) \\
85(93.0)\end{array}$ \\
\hline 12 & 7.9 & $\begin{array}{l}\text { Apigenin-6-C- } \\
\text { hexoside-8-C- } \\
\text { rhamnoside }\end{array}$ & $224,272,313$ & 577 & $\begin{array}{l}\mathrm{MS}^{2}[577]: 487(41.2), 473(2.7), 457(100), 353(36.9) \\
\mathrm{MS}^{3}[577 \rightarrow 457]: 383(18.5), 354(33.0), 353(100) \\
\mathrm{MS}^{4}[577 \rightarrow 457 \rightarrow 353]: 326(55.5), 325(100), 298(22.0)\end{array}$ \\
\hline 13 & 8.3 & Apigenin-8-C-hexoside & $224,272,314$ & 431 & $\begin{array}{l}\operatorname{MS}^{2}[431]: 341(23.1), 311(100) \\
\operatorname{MS}^{3}[431 \rightarrow 311]: 284(38.1), 283(100) \\
\operatorname{MS}^{4}[431 \rightarrow 311 \rightarrow 283]: 283(100), 224(77.3), 163(23.7)\end{array}$ \\
\hline 14 & 8.8 & $\begin{array}{l}\text { Quercetin-O- } \\
\text { dihexoside }\end{array}$ & - & 625 & $\begin{array}{l}\text { MS }^{2}[625]: 302(17.1), 301(100), 300(19.1) \\
\text { MS }^{3}[625 \rightarrow 301]: 271(22.9), 212(10.8), 179(82.9), 151(100) \\
\text { MS }^{4}[625 \rightarrow 301 \rightarrow 151]: 169(100), 107(20.2)\end{array}$ \\
\hline 15 & 9.1 & Luteolin-7-O-hexoside & - & 447 & $\begin{array}{l}\text { MS }^{2}[447]: 286(14.0), 285(100) \\
\text { MS3 [447 } \rightarrow 285]: 285 \text { (100), } 241 \text { (24.9), } 176(19.0), 175 \text { (80.1), } 149 \text { (117.5) }\end{array}$ \\
\hline 16 & 9.3 & $\begin{array}{l}\text { Mearnsetin-O- } \\
\text { hexoside }\end{array}$ & $257,300,342$ & 493 & $\begin{array}{l}\operatorname{MS}^{2}[493]: 332(19.0), 331(100), 330(10.3), 316(11.7) \\
\operatorname{MS}^{3}[493 \rightarrow 331]: 317(12.8), 316(100), 315(16.3) \\
\operatorname{MS}^{4}[493 \rightarrow 331 \rightarrow 316]: 287(49.6), 271(46.1), 229(38.2), 166(100)\end{array}$ \\
\hline $17^{*}$ & 9.5 & $\begin{array}{l}\text { Quercetin-3-O- } \\
\text { glucoside }\end{array}$ & 258,353 & 463 & $\begin{array}{l}\operatorname{MS}^{2}[463]: 302(17.1), 301(100), 300(19.1) \\
\text { MS }^{3}[463 \rightarrow 301]: 271(22.9), 212(10.8), 179(82.9), 151(100) \\
\text { MS }^{4}[463 \rightarrow 301 \rightarrow 151]: 169(100), 107(20.2)\end{array}$ \\
\hline 18 & 9.8 & $\begin{array}{l}\text { Isorhamnetin-O- } \\
\text { hexoside }\end{array}$ & $256,270,344$ & 477 & $\operatorname{MS}^{2}$ [477]: 316 (17.1), 315 (100), 300 (29.3) \\
\hline
\end{tabular}


Table 2 (Continued)

\begin{tabular}{|c|c|c|c|c|c|}
\hline No. & $t_{\mathrm{R}}(\min )$ & Identification & $\lambda_{\max }(\mathrm{nm})$ & {$[\mathrm{M}-\mathrm{H}]^{-}(m / z)$} & HPLC-DAD-ESI/MS ${ }^{\mathrm{n}} \mathrm{m} / z$ (\% base peak) \\
\hline & & & & & $\begin{array}{l}\operatorname{MS}^{3}[477 \rightarrow 315]: 301(11.4), 300(100) \\
\operatorname{MS}^{4}[477 \rightarrow 315 \rightarrow 300]: 284(62.1), 271(67.5), 245 \text { (63.1), } 229(100), 213 \\
(61.9)\end{array}$ \\
\hline $19^{*}$ & 11.8 & $\begin{array}{l}\text { 3,4-O-Dicaffeoylquinic } \\
\text { acid }\end{array}$ & $246,299,325$ & 515 & $\begin{array}{l}\mathrm{MS}^{2}[515]: 354(17.5), 353(100), 335(12.3), 299(12.2), 173(21.8) \\
M^{3}[515 \rightarrow 353]: 191(24.8), 179(60.9), 173(100), 135(14.8) \\
M^{4}[515 \rightarrow 353 \rightarrow 173]: 155(20.8), 111(100), 93(61.1)\end{array}$ \\
\hline $20^{*}$ & 12.5 & $\begin{array}{l}\text { 1,5-O-Dicaffeoylquinic } \\
\text { acid }\end{array}$ & $243,300,328$ & 515 & $\begin{array}{l}\operatorname{MS}^{2}[515]: 353(100) \\
\operatorname{MS}^{3}[515 \rightarrow 353]: 191(100) \\
\operatorname{MS}^{4}[515 \rightarrow 353 \rightarrow 191]: 173(43.8), 127(100), 109(22.1)\end{array}$ \\
\hline $21^{*}$ & 12.9 & $\begin{array}{l}\text { 3,5-O-Dicaffeoylquinic } \\
\text { acid }\end{array}$ & $242,300,328$ & 515 & $\begin{array}{l}\operatorname{MS}^{2}[515]: 354(10.7), 353(100) \\
\operatorname{MS}^{3}[515 \rightarrow 353]: 191(100), 179(53.9) \\
\operatorname{MS}^{4}[515 \rightarrow 353 \rightarrow 191]: 173(100), 127(90.9), 93(34.5)\end{array}$ \\
\hline 22 & 13.1 & $\begin{array}{l}\text { Dicaffeoylquinic acid } \\
\text { isomer }\end{array}$ & 240,326 & 515 & $\begin{array}{l}\text { MS }^{2}[515]: 354(15.8), 353(100) \\
\text { MS }^{3}[515 \rightarrow 353]: 191(100), 179(52.1), 135(15.2) \\
\text { MS }^{4}[515 \rightarrow 353 \rightarrow 191]: 85(100), 173(76.6), 127(66.8), 109(76.6)\end{array}$ \\
\hline $23^{*}$ & 14.2 & $\begin{array}{l}\text { 4,5-O-dicaffeoylquinic } \\
\text { acid }\end{array}$ & $243,300,327$ & 515 & $\begin{array}{l}\operatorname{MS}^{2}[515]: 354(14.5), 353(100) \\
\text { MS }^{3}[515 \rightarrow 353]: 191(31.5), 179(53.9), 173(100) \\
\operatorname{MS}^{4}[515 \rightarrow 353 \rightarrow 179]: 155(33.6), 111(57.0), 93(100)\end{array}$ \\
\hline 24 & 14.6 & Luteolin-7-O-pentoside & & 417 & $\begin{array}{l}\mathrm{MS}^{2} \text { [417]: } 285 \text { (100), } 284(22.4) \\
\left.\mathrm{MS}^{3} \text { [417 } \rightarrow 285\right]: 257(5.1), 243(72.1), 241 \text { (54.1), } 217 \text { (93.1), } 199 \text { (98.2), } \\
175 \text { (100), } 151 \text { (39.2) }\end{array}$ \\
\hline 25 & 15.3 & $\begin{array}{l}\text { Dihydroxy- } \\
\text { dimethoxyl-O- } \\
\text { hexoside } \\
\text { flavone }\end{array}$ & & 491 & $\begin{array}{l}\text { MS }^{3}[491 \rightarrow 329]: 315(21.1), 314(100) \\
\text { MS }^{4}[491 \rightarrow 329 \rightarrow 314]: 300(22.8), 299(100)\end{array}$ \\
\hline 26 & 16.8 & $\begin{array}{l}\text { 3-p-O-Coumaroyl-5-O- } \\
\text { caffeoylquinic } \\
\text { acid }\end{array}$ & & 499 & 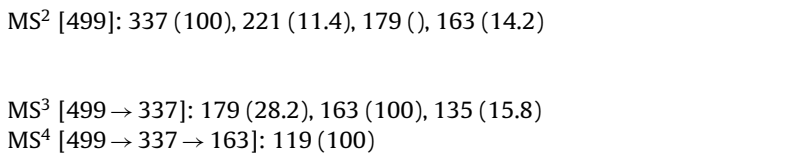 \\
\hline 27 & 18.1 & Unknown & & 423 & $\begin{array}{l}\text { MS }^{2}[423]: 262(10.6), 261(100), 173(100) \\
\text { MS }^{3}[423 \rightarrow 261]: 175(11.6), 173(100) \\
\text { MS }^{4}[423 \rightarrow 261 \rightarrow 173]: 93(100)\end{array}$ \\
\hline 28 & 18.9 & $\begin{array}{l}1-O-\text { Caffeoyl-5-O- } \\
\text { feruloylquinic } \\
\text { acid }\end{array}$ & 328 & 529 & $\begin{array}{l}\text { MS }^{2}[529]: 368(12.9), 367(100), 353(30.0), 191(8.5) \\
\text { MS }^{3}[529 \rightarrow 367]: 191(100) \\
\operatorname{MS}^{4}[529 \rightarrow 367 \rightarrow 191]: 173(69.0), 127(100), 109(52.9)\end{array}$ \\
\hline 29 & 20.2 & $\begin{array}{l}1 \text { or } 5-O \text {-caffeoyl- } 4-O- \\
\text { feruloylquinic } \\
\text { acid }\end{array}$ & - & 529 & 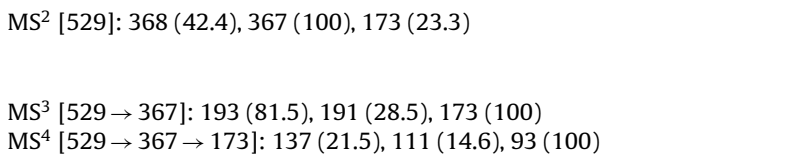 \\
\hline 30 & 20.6 & $\begin{array}{l}\text { 3-O-feruloyl-5-O- } \\
\text { caffeoylquinic } \\
\text { acid }\end{array}$ & & 529 & 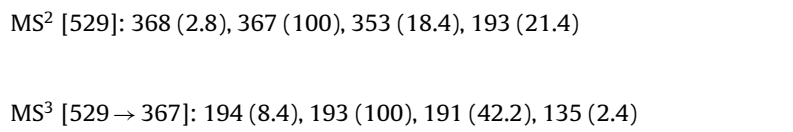 \\
\hline 31 & 22.2 & $\begin{array}{l}\text { 3,4-O-diferuloylquinic } \\
\text { acid }\end{array}$ & & 543 & 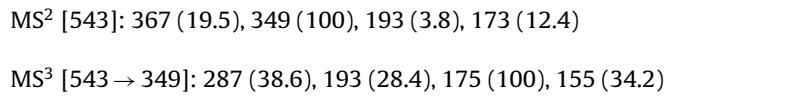 \\
\hline 32 & 23.2 & $\begin{array}{l}\text { 3,5-O-diferuloylquinic } \\
\text { acid }\end{array}$ & & 543 & $\begin{array}{l}\text { MS }^{2} \text { [543]: } 367(100), 349(38.2), 191(18.28) \\
\text { MS }^{3}[543 \rightarrow 367]: 193(100), 173(2.4)\end{array}$ \\
\hline 33 & 23.8 & $\begin{array}{l}\text { Kaempferol-3-O- } \\
\text { caffeoylhexoside }\end{array}$ & - & 609 & 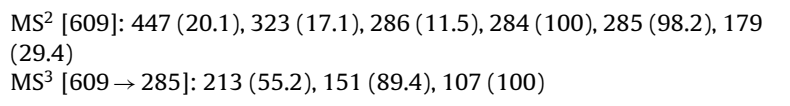 \\
\hline 34 & 26.5 & $\begin{array}{l}\text { Quercetin-O- } \\
\text { caffeoylhexoside }\end{array}$ & 253,330 & 625 & $\begin{array}{l}\mathrm{MS}^{2}[625]: 463(57.1), 445(24.2), 323(14.3) \\
301(100), 300(5.1) \\
\text { MS }^{3}[625 \rightarrow 301]: 273(4.1), 271(5.9), 255(19.6)\end{array}$ \\
\hline
\end{tabular}


Table 2 (Continued)

\begin{tabular}{|c|c|c|c|c|c|}
\hline No. & $t_{\mathrm{R}}(\min )$ & Identification & $\lambda_{\max }(\mathrm{nm})$ & {$[\mathrm{M}-\mathrm{H}]^{-}(m / z)$} & HPLC-DAD-ESI/MS ${ }^{n} m / z$ (\% base peak) \\
\hline & & & & & $\begin{array}{l}179(45.9), 151(100), 107(7.0) \\
\operatorname{MS}^{4}[625 \rightarrow 301 \rightarrow 151]: 107(100)\end{array}$ \\
\hline 35 & 27.2 & Unknown & & 579 & $\begin{array}{l}\operatorname{MS}^{2}[579]: 418(24.7), 417(100) \\
\text { MS }^{3}[579 \rightarrow 417]: 243(100), 179(26.5) \\
\text { MS }^{4}[579 \rightarrow 417 \rightarrow 243]: 225(16.7), 199(100), 183(31.0)\end{array}$ \\
\hline $36^{*}$ & 27.7 & Luteolin & - & 285 & $\begin{array}{l}\text { MS }^{2}[285]: 243(38.2), 241(92.1), 217(12.5), 199(69.5), 175 \text { (100), } 151 \\
(40.2) \\
\text { MS }^{3}[285 \rightarrow 175]: 147(100)\end{array}$ \\
\hline 37 & 28.5 & $\begin{array}{l}\text { Caffeoylcoumaroyltartaric } \\
\text { acid }\end{array}$ & $232,300,311$ & 457 & $\begin{array}{l}\text { MS }^{2}[457]: 296(10.5), 295(100), 173(12.9) \\
\text { MS }^{3}[457 \rightarrow 295]: 163(100), 121(36.2) \\
\text { MS }^{4}[457 \rightarrow 295 \rightarrow 163]: 111(38.8), 93(100)\end{array}$ \\
\hline $38^{*}$ & 29.2 & $\begin{array}{l}3,4,5-O- \\
\text { Tricaffeoylquinic } \\
\text { acid }\end{array}$ & & 677 & $\mathrm{MS}^{2}$ [457]: 296 (10.5), 295 (100), 173 (12.9) \\
\hline & & & & & $\begin{array}{l}\operatorname{MS}^{3}[457 \rightarrow 295]: 163(100), 121(36.2) \\
\text { MS }^{4}[457 \rightarrow 295 \rightarrow 163]: 111(38.8), 93(100)\end{array}$ \\
\hline 39 & 30.4 & $\begin{array}{l}\text { Eriodictyol-7-O- } \\
\text { hexoside }\end{array}$ & 247,329 & 449 & $\begin{array}{l}\operatorname{MS}^{2}[449]: 287(100), 173(14.3) \\
\operatorname{MS}^{3}[449 \rightarrow 287]: 173(100) \\
\text { MS }^{4}[449 \rightarrow 287 \rightarrow 173]: 111(100), 83(73.6)\end{array}$ \\
\hline $40^{*}$ & 33.9 & Apigenin & 260,331 & 269 & $\begin{array}{l}\text { MS }^{2} \text { [269]: } 227(33.4), 225(100), 201(59.2), 151(32.6), 149(80.3) \\
\text { MS }^{3}[269 \rightarrow 225]: 198(23.1), 183 \text { (66.9), } 181(100)\end{array}$ \\
\hline
\end{tabular}

(-) Their UV spectra have not been properly observed due to low intensity.

* Comparison with reference standards.

Hydroxycinnamic acid derivatives<smiles>[R9]OC1CCC(O)(C(=O)O)CC(O)C1O[Na]</smiles>

Flavonoids<smiles>O=c1cc(-c2ccc(O)c(O)c2)oc2cc(O)cc(O)c12</smiles>

Luteolin

Isorhamnetin<smiles>COc1ccc(-c2oc3cc(O)cc(O)c3c(=O)c2O)cc1O</smiles><smiles>[R]c1cc(/C=C/C(C)=O)ccc1O</smiles>

R: Caffeoyl $\mathrm{OH}$ Feruoyl $\mathbf{O C H}_{3}$ Coumaroyl $\mathrm{H}$<smiles>[R]C1OC(O)C(O)C(O)C1O</smiles>

R:

Hexosyl $\quad \mathrm{CH}_{2} \mathrm{OH}$

Rhamnosyl $\mathrm{CH}_{3}$<smiles>O=c1cc(-c2ccc(O)cc2)oc2cc(O)cc(O)c12</smiles>

Apigenin<smiles>O=c1c(O)c(-c2ccc(O)c(O)c2)oc2cc(O)cc(O)c12</smiles>

Quercetin<smiles>O=c1c(O)c(-c2cccc(O)c2)oc2cc(O)cc(O)c12</smiles>

Kaempferol<smiles>COc1c(O)cc(-c2oc3cc(O)cc(O)c3c(=O)c2O)cc1O</smiles>

Mearnsetin

Fig. 2. Chemical structures of phenolic compounds characterized. 
Compounds $\mathbf{9}\left(t_{\mathrm{R}}=6.5 \mathrm{~min}\right)$ and $\mathbf{1 2}\left(t_{\mathrm{R}}=7.9 \mathrm{~min}\right)$ displayed a [M$\mathrm{H}]^{-}$ion at $\mathrm{m} / \mathrm{z} 577 . \mathrm{MS}^{\mathrm{n}}$ experiments of these two peaks did not revealed the fragment corresponding to the neutral loss of $60 \mathrm{Da}$ and the presence of a fragment ion at $m / z 353$ indicates that the sugar groups must be a hexoside or a methylpentose.

Compound 9 presented $\mathrm{MS}^{2}$ ions at $m / z 559$ (21.9\% of base peak) $\left[\mathrm{M}-\mathrm{H}-\mathrm{H}_{2} \mathrm{O}\right]^{-}$and $m / z 503[\mathrm{M}-\mathrm{H}-74]^{-}$suggests that the rhamnoside group should be linked at 6 -C-position. Thus, $\mathbf{9}$ was identified as apigenin-6-C-rhamnoside-8- $C$-hexoside.

Compound 12 has been reported before and was identified as apigenin-6-C-hexoside-8- $C$-rhamnoside.

Compound $13\left(t_{\mathrm{R}}=8.3 \mathrm{~min}\right)$ showed a $[\mathrm{M}-\mathrm{H}]^{-}$ion at $\mathrm{m} / \mathrm{z} 431$. The $\mathrm{MS}^{2}$ spectrum presented main fragment ions at $\mathrm{m} / z 311$ [M$\mathrm{H}-120]^{-0,2} \mathrm{X}^{-}$(base peak) and $341{ }^{0,3} \mathrm{X}^{-}$(23.1\% of base peak). The loss of water molecules is indicative of C-6 isomers. This type of cleavage was not observed for compound 13 and based on literature reports (Gouveia S. and Castilho) this compound was identified as apigenin-8-C-hexoside.

3.2.1.3. O-glycosides $(3,14,15,16,17,18,24,25,33$ and 34). The fragments resulted from the of $O$-glycosilated flavonoids were labelled as proposed by Cuyckens and Claeys (2004). The ${ }^{i, j} A^{-}$and ${ }^{i, j} B^{-}$labels correspond to ions containing intact A- and B-rings, respectively, and $i$ and $j$ specify the $\mathrm{C}$-ring bonds that have been broken.

Compound $3\left(t_{\mathrm{R}}=4.0 \mathrm{~min}\right)$ displayed a $[\mathrm{M}-\mathrm{H}]^{-}$ion at $m / z 609$. Its analysis by $\mathrm{MS}^{2}$ fragmentation resulted in a neutral loss of $162 \mathrm{Da}$ forming a fragment ion at $\mathrm{m} / \mathrm{z} 447$ and an intense fragment at $\mathrm{m} / \mathrm{z}$ 285 (34.9\% of base peak).

MS $^{3}$ spectrum displayed a fragment ion at $\mathrm{m} / \mathrm{z} 285$ (loss of $162 \mathrm{Da})$, as base peak, and its subsequent fragmentation gave the typical fragments of kaempferol at $m / z 255\left[\mathrm{Y}_{0}{ }^{-}-\mathrm{CH}_{2} \mathrm{OH}\right]^{-}$ and $151\left({ }^{1,3} A^{-}\right)$(comparison made with a standard solution of kaempferol). The absence of a fragment ion at $m / z 323$ excluded the hypothesis of the two 162 Da residues being caffeoylhexosides and were characterized as being two hexosides residues. Based on the previous reports (Gouveia and Castilho, 2010) and according to the rules described by Ablajan and co-workers (Ablajan et al., 2006) 3 was identified as kaempferol-3,7-Odihexoside.

Compound $33\left(t_{\mathrm{R}}=23.8 \mathrm{~min}\right)$ also displayed a $[\mathrm{M}-\mathrm{H}]^{-}$ion at $\mathrm{m} / \mathrm{z}$ 609 . However, $\mathrm{MS}^{\mathrm{n}}$ fragmentation was quite different of that found for compound 4. The MS ${ }^{2}$ spectra showed the radical aglycone ion $\left[\mathrm{Y}_{0}-\mathrm{H}\right]^{-}$at $m / z 284$, as base peak, and also an intense fragment at $\mathrm{m} / z 285$ ( $98.2 \%$ of base peak).

The loss of a $324 \mathrm{Da}$ residue was attributed to a combined loss of two 162 Da groups and was confirmed by the presence of a fragment ion at $\mathrm{m} / \mathrm{z} 447$ (20.1\% of base peak) formed by the loss of $162 \mathrm{Da}$. The fragment ion at $\mathrm{m} / \mathrm{z} 323$ ( $17.1 \%$ of base peak) assigned as [caffeoylhexoside- $\mathrm{H}]^{-}$point out to a hexoside group esterified with a caffeoyl group rather than two hexosides moieties. The aglycone was identified as being kaempferol based on the $\mathrm{MS}^{\mathrm{n}}$ fragments and comparison with a kaempferol reference solution.

The favoured glycosilation positions for flavonols, such as kaempferol are 3-OH and 7-OH. When the aglycone radical is more abundant than the deprotonated aglycone ion, indicates an aglycone substituted at position 3-OH (Cuyckens and Claeys, 2005). For compound 33, the $\mathrm{MS}^{2}$ spectrum base peak was the aglycone radical ion, at $m / z 285$, and therefore it was identified as being kaempferol-3-O-caffeoylhexoside.

Compound $17\left(t_{\mathrm{R}}=9.5 \mathrm{~min}\right)$ exhibited a $[\mathrm{M}-\mathrm{H}]^{-}$ion at $\mathrm{m} / \mathrm{z} 463$ which easily lost a 162 Da moiety, in $\mathrm{MS}^{2}$ fragmentation, resulting in a fragment ion at $m / z 301$. Further fragmentation of this ion gave the representative fragments of quercetin at $m / z 151\left({ }^{1,2} \mathrm{~A}^{-}-\mathrm{CO}\right)$, $179\left(\left[{ }^{1,2} \mathrm{~A}^{-}-\mathrm{H}\right]^{-}\right)$and $271\left[\mathrm{M}-\mathrm{H}-\mathrm{CH}_{2} \mathrm{O}\right]^{-}$. Therefore, compound
17 was unequivocally identified as quercetin-3-O-glucoside by comparison with a reference standard solution.

Compounds $14\left(t_{R}=8.8 \mathrm{~min}\right)$ and $34\left(t_{R}=26.5 \mathrm{~min}\right)$ gave the same $[\mathrm{M}-\mathrm{H}]^{-}$ion at $m / z 625$ and their $\mathrm{MS}^{\mathrm{n}}$ fragmentation was similar. In the $\mathrm{MS}^{2}$ spectra, the same base peak at $\mathrm{m} / \mathrm{z} 301$ was observed (loss of $324 \mathrm{Da}$ ). The occurrence of a MS ${ }^{1}$ ion at $\mathrm{m} / z 463$ (loss of $162 \mathrm{Da}$ ) suggests that the residue of $324 \mathrm{Da}$ is composed of two units of $162 \mathrm{Da}$ linked. However, the nature of these two groups appears to be distinct for each compound. For 34, a MS ${ }^{1}$ ion at $m / z 323$ (14.3\% of base peak) was observed indicating a caffeoylhexoside group which is in good agreement with the long retention time of this compound. The lower retention time of $\mathbf{1 4}$ and the absence of a fragment ion at $m / z 323$ indicate a dihexoside residue.

Fragmentation of the deprotonated aglycone ion, $\mathrm{Y}_{0}^{-}$at $m / z$ 301 allowed to identify common fragment ions of quercetin, as described for compound $\mathbf{1 7}$.

Since the $\mathrm{Y}_{0}^{-}$ion is the $\mathrm{MS}^{2}$ base peak, the substitution groups must be linked to only one $\mathrm{OH}$ group of aglycone structure. The aglycone radical ion was not observed, so the 3-OH position is excluded but no other fragments were found to support in which position the substitution occurs. Thus, 14 was tentatively identified as quercetin- $O$-dihexoside and $\mathbf{3 4}$ was as quercetin- $O$ caffeoylhexoside.

Compound $15\left(t_{\mathrm{R}}=9.1 \mathrm{~min}\right)$ displayed a $[\mathrm{M}-\mathrm{H}]^{-}$in at $m / z 447$ and its $\mathrm{MS}^{2}$ fragmentation revealed the loss of $162 \mathrm{Da}$ forming a fragment ion at $m / z 285, Y_{0}^{-}$. This compound was already described for Artemisia argentea (Gouveia and Castilho, 2011a, b) and identified as luteolin-7-O-hexoside.

Another luteolin derivative was detected at a retention time of 14.6 min (compound 24). It gave a $[\mathrm{M}-\mathrm{H}]^{-}$ion at $\mathrm{m} / \mathrm{z} 417$ easily lost a neutral group of $132 \mathrm{Da}$ (pentoside) rising the deprotonated aglycone ion at $m / z 285$. Fragmentation of the ion at $m / z 285$ gave the characteristics fragments of luteolin at $m / z 175\left(\left[\mathrm{M}-\mathrm{H}-\mathrm{C}_{3} \mathrm{O}_{2}-\right.\right.$ $\left.\left.\mathrm{C}_{2} \mathrm{H}_{2} \mathrm{O}\right]^{-}\right), 217\left(\left[\mathrm{M}-\mathrm{H}_{-} \mathrm{C}_{3} \mathrm{O}_{2}\right]^{-}\right)$and $241\left(\left[\mathrm{M}-\mathrm{H}-\mathrm{CO}_{2}\right]^{-}\right)$(Fig. 3). The favoured substitution position for flavones such as luteolin is the 7$\mathrm{OH}$ position (Cuyckens and Claeys, 2004). Consequently, compound 24 was characterized as luteolin 7-O-pentoside. To our knowledge it is the first time that this compound is reported for Artemisia species.

Compound $16\left(t_{\mathrm{R}}=9.3 \mathrm{~min}\right)$ showed a $[\mathrm{M}-\mathrm{H}]^{-}$ion at $m / z 493$. Its $\mathrm{MS}^{\mathrm{n}}$ fragmentation resulted in the aglycone ion at $m / z 331$ due to the loss of $162 \mathrm{Da}$. The $\mathrm{MS}^{3}$ radical ion at $m / z 316$ is similar to the fragmentation behaviour described for mearnsetin-O-hexoside, also detected in Artemisia argentea and Artemisia annua (Gouveia and Castilho, 2011a,b; Han et al., 2008).

Compound $18\left(t_{\mathrm{R}}=9.8 \mathrm{~min}\right)$ displayed a $[\mathrm{M}-\mathrm{H}]^{-}$ion at $\mathrm{m} / z 477$ and was characterized as isorhamnetin- $O$-hexoside by comparison with literature data (Gouveia and Castilho, 2009). This compound was not reported before for Artemisia annua but was detected in Artemisia species (Gouveia and Castilho, 2011a,b).

Compound $25\left(t_{\mathrm{R}}=15.3 \mathrm{~min}\right)$ showed a $[\mathrm{M}-\mathrm{H}]^{-}$ion at $\mathrm{m} / \mathrm{z}$ 491. A loss of $162 \mathrm{Da}$ was observed in the $\mathrm{MS}^{2}$ fragmentation, forming a fragment ion at $\mathrm{m} / \mathrm{z}$ 329. In the further $\mathrm{MS}^{\mathrm{n}}$ fragmentations, two losses of $15 \mathrm{Da}$ each were observed and associated to two methoxyl groups. So, 25 was characterized as dihydroxy-dimethoxyl-O-hexoside flavones. This compound was also reported for Artemisia argentea (Gouveia and Castilho, 2011a,b) but it was not reported for Artemisia annua before.

One compound (39) from the group of flavanones is reported for Artemisia annua for the first time. It was found at a retention time of $30.4 \mathrm{~min}$ and identified as a eriodictyol-7-O-hexoside. The [M$\mathrm{H}]^{-}$ion appeared at $\mathrm{m} / \mathrm{z} 449$ and the hexoside residue was easily expelled in the MS ${ }^{2}$ fragmentation resulting in the deprotonated aglycone ion, $\mathrm{Y}_{0}^{-}$, at $m / z 287$. 


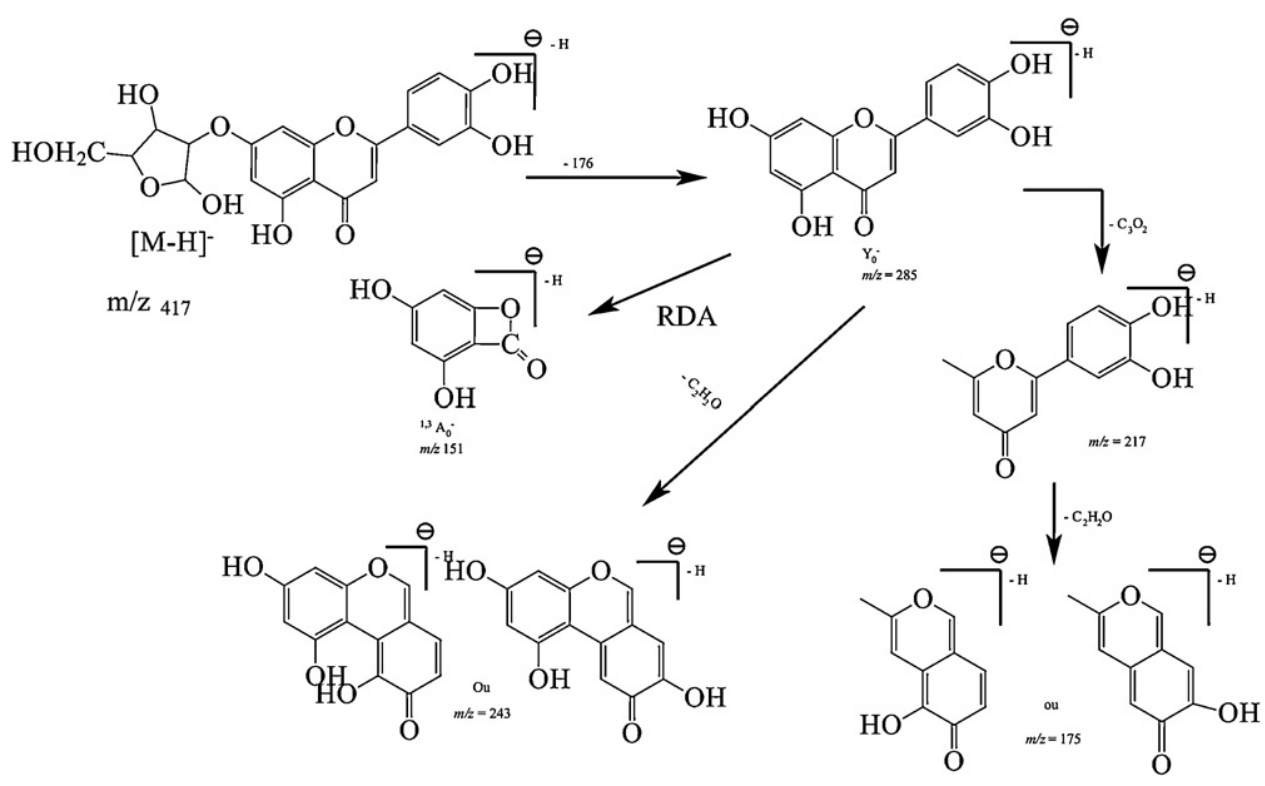

Fig. 3. Proposed fragmentation pathway for compound 24-luteolin-O-pentoside.

\subsubsection{Identification of hydroxycinnamic acids $(\mathbf{2}, \mathbf{4}, \mathbf{5}, \mathbf{6}, \mathbf{1 0}, \mathbf{1 1}$,}

\section{$19,20,21,22,23,26,28,29,30,31,32$ and 38 )}

Compound $2\left(t_{\mathrm{R}}=3.1 \mathrm{~min}\right)$ displayed a $[\mathrm{M}-\mathrm{H}]^{-}$ion at $\mathrm{m} / \mathrm{z} 191$ and was identified as quinic acid (Gouveia and Castilho, 2011a,b). Despite that this compound was found as a relative intense component of the Artemisia annua extract, it was not reported before for this plant.

A total of 19 quinic acid derivatives were detected and identified in this study, most of them quinic acid esterified with acyl groups. The identification of each compound was made based on the main fragment ions obtained in the MS ${ }^{\mathrm{n}}$ experiments. The hierarchical key for the identification by LC-MS ${ }^{\mathrm{n}}$ of quinic acid derivatives proposed by Clifford et al. (Clifford et al., 2003, 2005) was used to identify this class of compounds.

Mono-, di- and tricaffeoylquinic acids were identified by comparison of the retention time, $\mathrm{MS}^{\mathrm{n}}$ fragmentation behaviour and UV spectra with those of standard compounds. It was the case of compounds $\mathbf{5}\left(t_{\mathrm{R}}=4.4 \mathrm{~min}\right)$ as 5- $O$-caffeoylquinic acid; $\mathbf{1 9}\left(t_{\mathrm{R}}=11.8 \mathrm{~min}\right)$ as 3,4-O-dicaffeoylquinic acid; $20\left(t_{\mathrm{R}}=12.5 \mathrm{~min}\right)$ as $1,5-\mathrm{O}$ dicaffeoylquinic acid; 21 ( $t_{\mathrm{R}}=12.9 \mathrm{~min}$ ) as 3,5-O-dicaffeoylquinic acid; $23\left(t_{\mathrm{R}}=14.2 \mathrm{~min}\right)$ as 4,5-O-dicaffeoylquinic acid and $\mathbf{3 8}$ $\left(t_{\mathrm{R}}=29.2 \mathrm{~min}\right)$ as 3,4,5-O-tricaffeoylquinic acid.

Additionally, two monocaffeoylquinic acids, compounds $\mathbf{4}$ and 6, were identified based on $\mathrm{MS}^{\mathrm{n}}$ fragmentation of the $[\mathrm{M}-\mathrm{H}]^{-}$ion at $m / z$ 353. Compound $4\left(t_{\mathrm{R}}=4.4 \mathrm{~min}\right)$ gave a $\mathrm{MS}^{2}$ ion at $\mathrm{m} / z 191$ (base peak) and an intense fragment ion at $m / z 179$ (51.4\% of base peak) which is representative of a caffeoyl group linked to the 3-OH position of quinic acid. Thus, 4 was identified as 3-O-caffeoyquinic acid.

Compound $6\left(t_{R}=5.2 \mathrm{~min}\right.$ ) showed a MS ${ }^{2}$ ion at $\mathrm{m} / z 173$ (base peak) pointing to a quinic acid esterified at position 4-OH (Fig. 4). Therefore, 6 was classified as 4-O-caffeoylquinic acid. Remarkably, this compound gave a higher intensity than the isomer 5-O-CQA commonly found in other Asteraceae plants.

Compound $22\left(t_{\mathrm{R}}=13.1 \mathrm{~min}\right)$ gave a $[\mathrm{M}-\mathrm{H}]^{-}$ion at $m / z 515$ and further $\mathrm{MS}^{\mathrm{n}}$ fragmentation gave the characteristic fragment ions of dicaffeoylquinic acid isomers at $m / z 191,173$ and 353 . This should be a cis isomer of a dicaffeoylquinic acid with no substitution position 4-OH (Jaiswal et al., 2011; Ma et al., 2008). The unequivocally identification of this compound can only be established by NRM studies or comparison with a reference standard since even
UV-irradiation studies would not clarify if it is a mono-cis or a di-cis compound.

Compounds $10\left(t_{R}=6.9 \mathrm{~min}\right)$ and $11\left(t_{R}=7.4 \mathrm{~min}\right)$ exhibited the same $[\mathrm{M}-\mathrm{H}]^{-}$ion at $m / z 367$ but a different $\mathrm{MS}^{\mathrm{n}}$ fragmentation behaviour. For 10, the $\mathrm{MS}^{2}$ spectrum showed a fragment ion at $m / z 193$ (base peak). Based on the rules reported by Clifford et al. (Clifford et al., 2003) this compound was identified as 3-Oferuloylquinic acid. Compound $\mathbf{1 1}$ gave, as base peak of the MS ${ }^{2}$ spectrum, a fragment ion at $m / z 191$ [quinic acid-H] ${ }^{-}$. This pattern is consistent to that reported for 5-O-feruloylquinic acid (Clifford et al., 2005).

Compound $26\left(t_{\mathrm{R}}=16.8 \mathrm{~min}\right)$ displayed a $[\mathrm{M}-\mathrm{H}]^{-}$ion at $\mathrm{m} / z 499$. The MS ${ }^{2}$ spectrum showed a fragment ion at $m / z 337$, as base peak, indicating the loss of $162 \mathrm{Da}$ and suggesting a coumaroylquinic acid derivative. $\mathrm{MS}^{3}$ fragmentation of the ion at $\mathrm{m} / \mathrm{z} 337$ resulted in a fragment ion at $m / z 163$ and a MS 4 ion at $m / z 119$, as base peaks, pointing to a $3-p-O$-coumaroylquinic acid structure, according to literature reports (Clifford et al., 2003).

The absence of a strong fragmentation at $m / z 173$ suggests a 3,5-O-p-coumaroyl-caffeoylquinic acid.

Since the caffeoyl group is the first to be lost it should be linked to the $5-\mathrm{OH}$ position. Taking into account these data, $\mathbf{2 6}$ was identified as 3-p-O-coumaroyl-5-O-caffeoylquinic acid.

To our knowledge, quinic acids esterified with coumaroyl groups have not been reported before in phenolic screenings for Artemisia annua.

Three compounds with $[\mathrm{M}-\mathrm{H}]^{-}$ions at $\mathrm{m} / \mathrm{z} 529$ were observed (compound $\mathbf{2 8}\left(t_{\mathrm{R}}=18.9 \mathrm{~min}\right), \mathbf{2 9}\left(t_{\mathrm{R}}=20.2 \mathrm{~min}\right)$ and $\mathbf{3 0}$ $\left.\left(t_{\mathrm{R}}=20.7 \mathrm{~min}\right)\right)$. In the $\mathrm{MS}^{2}$ fragmentation all compounds gave $\mathrm{MS}^{2}$ base peaks at $m / z 367$ [feruloylquinic acid-H] ${ }^{-}$, due to the loss of a caffeoyl residue, and a secondary fragment ion at $\mathrm{m} / \mathrm{z} 353$ (ca. 20\% of base peak). The distinction of these three isomers was based on the $\mathrm{MS}^{3}$ fragmentation.

Compound 28 gave a $\mathrm{MS}^{3}$ ion at $m / z 191$, as base peak, which is consistent with a $5-O$-feruloylquinic acid structure. According to the fragmentation data, a fragment ion at $m / z 179$ was not observed, thus the caffeoyl group must be linked to the $1-\mathrm{OH}$ position of quinic acid. So, compound $\mathbf{2 8}$ was identified as 1-O-caffeoyl-5-Oferuloylquinic acid.

Compound $29\left(t_{R}=20.2 \mathrm{~min}\right)$ exhibited a $\mathrm{MS}^{3}$ base peak at $\mathrm{m} / \mathrm{z}$ 173 which indicates a 4-O-feruloylquinic acid structure. The exact 


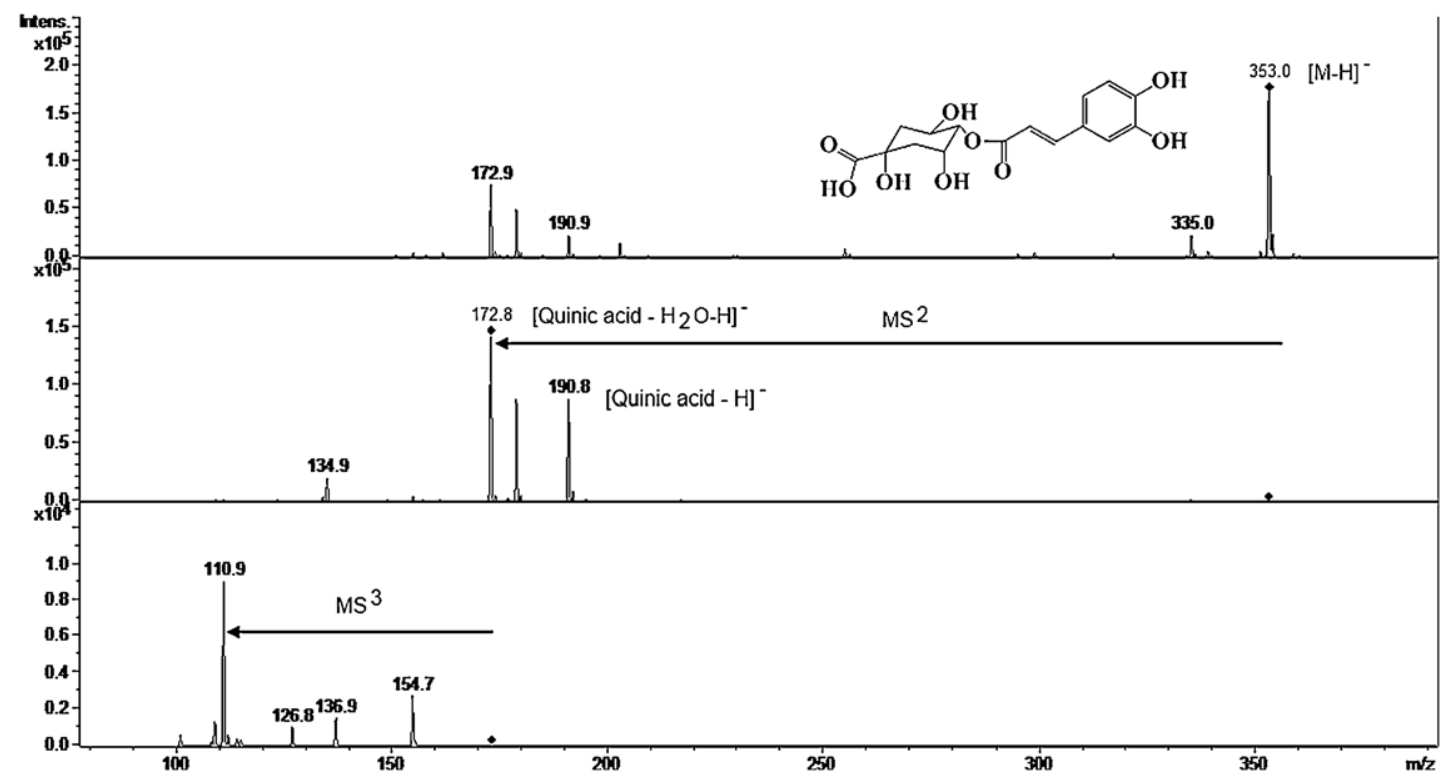

Fig. 4. Proposed fragmentation pathway for compound 6-4-O-caffeoylquinic acid.

location of the caffeoyl group is difficult to establish but since no fragment ion at $m / z 179$ was observed and given the presence of a weak fragment at $m / z 191$, the $3-\mathrm{OH}$ position is less probable. Therefore, compound 29 was characterized as 1 - or 5-O-caffeoyl4-O-feruloylquinic acid.

For compound 30 the $\mathrm{MS}^{3}$ ion at $m / z 193$ was the base peak which defines the presence of a feruloyl residue at the 3-OH position (Jaiswal et al., 2010).

According to the hierarchal key proposed by Clifford and coworkers (Clifford et al., 2003) this compound can be assigned as 3-O-feruloyl-5-O-caffeoylquinic acid.

Compounds $31\left(t_{R}=22.2 \mathrm{~min}\right)$ and $32\left(t_{R}=23.2 \mathrm{~min}\right)$ showed the same $[\mathrm{M}-\mathrm{H}]^{-}$ion at $m / z 543$. For compound 31, the $\mathrm{MS}^{2}$ spectrum gave a fragment ion at $m / z 349$ [M-H-ferulic acid- $\left.\mathrm{H}_{2} \mathrm{O}\right]^{-}$and further fragmentation was consistent with 3,4-O-diferuloylquinic acid as described in literature (Clifford et al., 2006). Compound 32 gave a $\mathrm{MS}^{2}$ base peak at $\mathrm{m} / \mathrm{z} 367$ [M-H-ferulic acid] ${ }^{-}$and was characterized as 3,5-O-diferuloylquinic acid (Clifford et al., 2006).

Compound $37\left(t_{\mathrm{R}}=28.5 \mathrm{~min}\right)$ showed a $[\mathrm{M}-\mathrm{H}]^{-}$ion at $m / z 457$ and was identified as a caffeoylcoumaroyltartaric acid isomer. This compound was also found in A. argentea (Gouveia and Castilho, 2011a, b).

Compound $1\left(t_{\mathrm{R}}=2.8 \mathrm{~min}\right)$ gave a $[\mathrm{M}-\mathrm{H}]^{-}$ion at $\mathrm{m} / \mathrm{z} 473$ was identified as a caffeic acid hexoside derivative. The caffeic acid moiety was identified by the $\mathrm{MS}^{2}$ ion at $m / z 179$.

\subsection{Antioxidant activity assays}

In complex samples such as EO and extracts even compounds present at low levels can present a relevant biological effect. For these reasons, the antioxidant capacity of these samples was evaluated.

The results obtained for the studied essential oils from three locations are shown in Table 3.

The DPPH and ABTS results showed that the EO from Jardim Botânico has a lower antiradical scavenging capacity (RSC) than EO from Preces and Porto Santo. These two EO were very similar and were not statistically different $(p=0.52)$.

All EO samples have similar reducing properties (ca. $1 \times 10^{7} \mathrm{mmol} \mathrm{FeSO}_{4} \cdot 7_{2} \mathrm{O} / \mathrm{mL}$ EO) since the FRAP results were not statistically different $(p>0.05)$.
In a recent study, our group analysed EO from thymol rich samples of oregano, known by its good antioxidant properties (Castilho et al., 2012), by the same DPPH method herein described. The Artemisia annua EO has a highest RSC than EO of oregano samples.

Comparison with other studies that measured the antioxidant capacity of Artemisia annua EO is difficult to establish given the different working protocols and forms to express the results. A recent article ('́avar et al., 2012) described the antioxidant capacity of EO of wild Artemisia annua and, like our results, the EO has a higher capacity to react to $\mathrm{ABTS}^{\circ+}$ cation radical rather than the DPPH radical but much weaker than thymol.

Radical-scavenging and reducing power ability of essential oils are usually associated with a high contents in aromatic components such as thymol, carvacrol and similar compounds, thus thymol is the current reference.

In Artemisia essential oils this type of substances is generally present in very minute amounts, so they should not have the most relevant effect.

An unusual antioxidant behaviour of $\alpha$ - and $\gamma$-terpinene in protecting methyl linoleate, DNA, and erythrocyte was reported (Li and Liu, 2009). The obtained results implied that the nonconjugated diene shows higher antioxidant capacity than a conjugated diene. Kawaree et al. (2008) found relevant antioxidant activity in Thai plants rich in sesquiterpenes such as germacrene-D, transbeta-caryophyllene and beta-elemene.

The unusual high antioxidant activity observed in the present study deserved further investigation by evaluation of individual components and their artificial mixtures.

Given the diversified phenolic profile of acetone extract, as described by the HPLC-DAD-ESI/MS ${ }^{n}$, the Folin-Ciocalteu method was performed to measure the total phenolic content (TPC) (Table 4). This method is widely used to measure the total phenolic content in different samples and the chemistry of this assay is based on the transfer of electrons in alkaline medium from phenolic compounds and other reducing species to molybdenum, forming blue complexes that can be detected at 750-765 nm (Magalhães et al., 2008).

This method is non-specific to phenolic compounds because other non-phenolic compounds with reducing properties (ascorbic acid, $\mathrm{Cu}(\mathrm{I}), \mathrm{Fe}(\mathrm{II})$, sulfur dioxide, aromatic amines, etc.) can also react (Magalhães et al., 2008). However, a high correlation between 
Table 3

Antioxidant capacity of Artemisia annua EO from three different origins.

\begin{tabular}{|c|c|c|c|}
\hline Artemisia anпuа EO & DPPH $\mu$ mol eq. Trolox $/ \mathrm{mL}$ EO & ABTS $\mu$ mol eq. Trolox/mL EO & FRAP $\mathrm{mmol} \mathrm{FeSO} 4.7_{2} \mathrm{O} / \mathrm{mL}$ EO \\
\hline Preces & $10.9 \pm 0.45$ & $38.1 \pm 1.1$ & $1.28 \times 10^{7} \pm 4.1 \times 10^{5}$ \\
\hline Jardim Botânico & $7.71 \pm 0.40$ & $25.9 \pm 1.5$ & $1.06 \times 10^{7} \pm 8.1 \times 10^{5}$ \\
\hline Porto Santo & $10.9 \pm 0.55$ & $32.7 \pm 1.9$ & $1.14 \times 10^{7} \pm 7.8 \times 10^{5}$ \\
\hline
\end{tabular}

Table 4

Antioxidant capacity of Artemisia annua acetone and methanol extracts.

\begin{tabular}{|c|c|c|c|c|c|}
\hline \multirow[t]{2}{*}{ Sample } & & $\mathrm{DPPH}$ & ABTS & \multirow[t]{2}{*}{ FRAP mmol FeSO $4.7_{2} \mathrm{O} / \mathrm{mg}$ dried plant } & \multirow[t]{2}{*}{ TPC mg GAE/100 g dried plant } \\
\hline & & \multicolumn{2}{|c|}{$\mu \mathrm{mol}$ eq. Trolox $/ 100 \mathrm{~g}$ dried plant } & & \\
\hline Acetone & $\begin{array}{l}\text { Preces } \\
\text { Jardim Botânico } \\
\text { Porto Santo }\end{array}$ & $\begin{array}{l}493.0 \pm 0.64 \\
307.2 \pm 0.061 \\
408.9 \pm 0.53\end{array}$ & $\begin{aligned} 2197.3 & \pm 19.9 \\
604.9 & \pm 25.5 \\
959.1 & \pm 33.4\end{aligned}$ & $\begin{array}{r}1861.0 \pm 67.2 \\
403.1 \pm 25.5 \\
1414.11 \pm 59.9\end{array}$ & $\begin{array}{l}521.2 \pm 5.4 \\
331.0 \pm 2.0 \\
396.3 \pm 7.1\end{array}$ \\
\hline Methanol & Preces & $341.1 \pm 0.87$ & $477.0 \pm 0.46$ & $935.1 \pm 0.22$ & $384.1 \pm 6.7$ \\
\hline
\end{tabular}

the results obtained by this method and those obtained by other ET-based assays (FRAP, DPPH, ABTS, etc.) have been reported and consists on the main advantage of the Folin-Ciocalteu method. The TPC values varied from $331.0 \pm 2.0$ to $521.2 \pm 5.4 \mathrm{mg}$ GAE $/ 100 \mathrm{~g}$ of dried plant. The plants collected in Preces gave the highest TPC values followed by those from Porto Santo and finally those from Jardim Botânico.

A recent paper on the analysis of methanolic extracts of Artemisia species (Carvalho et al., 2011) found a TPC value $(220 \pm 2 \mathrm{mg} \mathrm{GAE} / 100 \mathrm{~g})$ much lower than what we report here for the same solvent, those for acetone extraction being even higher (Table 4).

The results obtained for the antioxidant activity revealed the same relative order of TPC assay. The extract from Preces was the most active followed by Porto Santo and Jardim Botânico (Table 4).

In the DPPH assay the results varied from $307.2 \pm 0.061$ to $493.0 \pm 0.64 \mu \mathrm{mol}$ eq. Trolox $/ 100 \mathrm{~g}$ dried plant.

The values found for ABTS assay were higher than those of DPPH due to differences in the sensitivity of these methods. ABTS assay is more sensitivity since it measures the antioxidant activity of both hydrophilic and lipophilic antioxidants.

The FRAP results expressed the antioxidant capacity of the extract based on its reducing properties. The results obtained for Artemisia annua varied from $403.1 \pm 25.5$ to $1861.0 \pm 62.2 \mathrm{mmol}$ $\mathrm{FeSO}_{4} \cdot 7 \mathrm{H}_{2} \mathrm{O} / \mathrm{mg}$ dried plant and indicate a moderate reducing capacity.

Even though these comparisons can only be considered as a generic approach, they corroborate that acetone is a good solvent for recovery of phenolic compounds.

Artemisia argentea displayed a stronger antioxidant capacity (Gouveia and Castilho, 2011a, b) which is in good agreement with the findings of (Carvalho et al., 2011) where Artemisia annua was one of the weakest antioxidant plants among six Artemisia species.

\section{Conclusion}

Artemisia annua plants of artemisinin rich hybrids $(\mathrm{CPQBA} \times \mathrm{POP})$ cultivated in Madeira Archipelago were studied in terms of the volatile and phenolic composition.

The essential oil was mainly composed by monoterpenes with camphor and 1,8-cineole as major components. The effect of the direct exposure to light during the drying process as well as differences in cultivation location were studied and resulted only in small quantitative variations in EO composition mainly in the more volatile compounds (which showed higher percentage in plants dried in the shade).

In the acetone extract, several hydroxycinnamic acid derivatives and glycosylated flavonoids were separated and characterized. For the first time, caffeoylquinic acids esterified with coumaroyl groups were reported for this plant.

The antioxidant capacity of the samples was established by three different assays. Essential oils gave an unusual strong antioxidant capacity, while acetone extracts showed a moderate antioxidant power, when compared to other Artemisia species. However, the acetone extract showed stronger more antioxidant properties than the methanol extract.

The quantification of the detected main phenolic compounds and its relationship to the total antioxidant of the extracts should constitute the further work with this plant.

\section{Acknowledgments}

S. Gouveia is grateful to Fundação para a Ciência e Tecnologia (FCT) for a Ph.D. grant SFRH/BD/24227/2005. The mass spectrometer used in this work is part of the Portuguese National Mass Spectrometry Network (Contract RNEMREDE/1508/REM/2005) and was purchased in the framework of the National Programme for Scientific Re-equipment, with funds from POCI 2010 (FEDER) and FCT. This research was supported by FCT with funds from the Portuguese Government (Project PEst-OE/QUI/UI0674/2011).

\section{References}

Ablajan, K., Abliz, Z., Shang, X.-Y., He, J.-M., Zhang, R.-P., Shi, J.-G., 2006. Structura characterization of flavonol 3,7-di-O-glycosides and determination of the glycosylation position by using negative ion electrospray ionization tandem mass spectrometry. J. Mass Spectrom. 41, 352-360.

Benzie, I.F.F., Strain, J.J., 1996. The Ferric Reducing Ability of Plasma (FRAP) as a measure of "Antioxidant Power": The FRAP assay. Anal. Biochem. 239, 70-76.

Bhakuni, R.S., Jain, D.C., Sharma, R.P., Kumar, S., 2001. Secondary metabolites of Artemisia annua and their biological activity. Curr. Sci. 80, 35-48.

Bora, K.S., Sharma, A., 2011. The genus Artemisia: A comprehensive review. Pharmac. Biol. 49, 101-109.

Brisibe, E.A., Umoren, U.E., Brisibe, F., Magalhäes, P.M., Ferreira, J.F.S., Luthria, D. Wu, X., Prior, R.L., 2009. Nutritional characterisation and antioxidant capacity of different tissues of Artemisia annua L. Food Chem. 115, 1240-1246.

Carbonara, T., Pascale, R., Argentieri, M.P., Papadia, P., Fanizzi, F.P., Villanova, L., Avato, P., 2012. Phytochemical analysis of a herbal tea from Artemisia annua L. J. Pharmaceut. Biomed. Anal. 62, 79-86.

Carvalho, I.S., Cavaco, T., Brodelius, M., 2011. Phenolic composition and antioxidant capacity of six Artemisia species. Ind. Crops Prod. 33, 382-388.

Castilho, P.C., Gouveia, S.C., Rodrigues, A.I., 2008. Quantification of artemisinin in Artemisia annua extracts by ${ }^{1} \mathrm{H}-\mathrm{NMR}$. Phytochem. Anal. 19, 329-334.

Castilho, P.C., Savluchinske-Feio, S., Weinhold, T.S., Gouveia, S.C., 2012. Evaluation of the antimicrobial and antioxidant activities of essential oils, extracts and their main components from oregano from Madeira Island, Portugal. Food Control. 23, 552-558.

Ćavar, S., Maksimović, M., Vidic, D., Parić, A., 2012. Chemical composition and antioxidant and antimicrobial activity of essential oil of Artemisia annua L. from Bosnia. Ind. Crops Prod. 37, 479-485.

Clifford, M.N., Johnston, K.L., Knight, S., Kuhnert, N., 2003. Hierarchical scheme for LC-MS ${ }^{\mathrm{n}}$ identification of chlorogenic acids. J. Agric. Food Chem. 51, 2900-2911. 
Clifford, M.N., Knight, S., Kuhnert, N., 2005. Discriminating between the six isomers

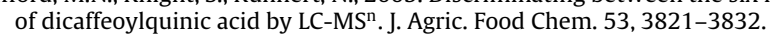

Clifford, M.N., Marks, S., Knight, S., Kuhnert, N., 2006. Characterization by LC-MS ${ }^{n}$ of four new classes of $p$-coumaric acid-containing diacyl chlorogenic acids in green coffee beans. J. Agric. Food Chem. 54, 4095-4101.

Cuyckens, F., Claeys, M., 2004. Mass spectrometry in the structural analysis of flavonoids. J. Mass Spectrom. 39, 1-15.

Cuyckens, F., Claeys, M., 2005. Determination of the glycosylation site in flavonoid mono-O-glycosides by collision-induced dissociation of electrospray-generated deprotonated and sodiated molecules. J. Mass Spectrom. 40, 364-372.

Ferreira, J.F.S., Luthria, D.L., Sasaki, T., Heyerick, A., 2010. Flavonoids from Artemisia annua L. as antioxidants and their potential synergism with artemisinin against malaria and cancer. Molecules 15, 3135-3170.

Gouveia, S.C., Castilho, P.C., 2012a. Antioxidant potential of Artemisia argentea L'Hér alcoholic extract and its relation with the phenolic composition. Food Res. Int. $45,362-368$.

Gouveia, S.C., Castilho, P.C., 2009. Analysis of phenolic compounds from different morphological parts of Helichrysum devium by liquid chromatography with on-line UV and electrospray ionization mass spectrometric detection. Rapid Commun. Mass Spectrom. 23, 3939-3953.

Gouveia, S.C., Castilho, P.C., 2010. Characterization of phenolic compounds in Helichrysum melaleucum by high-performance liquid chromatography with on-line ultraviolet and mass spectrometry detection. Rapid Commun. Mass Spectrom. 24, 1851-1868.

Gouveia, S.C., Castilho, P.C., 2011a. Antioxidant potential of Artemisia argentea L'Hér alcoholic extract and its relation with the phenolic composition. Food Res. Int. 44, 1620-1631.

Gouveia, S.C., Castilho, P.C., 2011b. Characterisation of phenolic acid derivatives and flavonoids from different morphological parts of Helichrysum obconicum by a RP-HPLC-DAD-(-)-ESI-MSn method. Food Chem. 129, 333-344.

Gouveia, S.C., Castilho, P.C., 2012b. Helichrysum monizii Lowe: Phenolic composition and antioxidant potential. Phytochem. Anal. 23, 72-83.

Han, J., Ye, M., Qiao, X., Xu, M., Wang, B.-r., Guo, D.-A., 2008. Characterization of phenolic compounds in the Chinese herbal drug Artemisia annua by liquid chromatography coupled to electrospray ionization mass spectrometry. J. Pharmaceut. Biomed. Anal. 47, 516-525.

Holm, Y., Laakso, I., Hiltunen, R., Galambosi, B., 1997. Variation in the essential oil composition of Artemisia annua L. of different origin cultivated in Finland. Flavour Frag. J. 12, 241-246.

Jaiswal, R., Kiprotich, J., Kuhnert, N., 2011. Determination of the hydroxycinnamate profile of 12 members of the Asteraceae family. Phytochemical 72, 781-790.

Jaiswal, R., Sovdat, T., Vivan, F., Kuhnert, N., 2010. Profiling and characterization by LC-MSn of the chlorogenic acids and hydroxycinnamoylshikimate esters in Mateǐ (Ilex paraguariensis). J. Agric. Food Chem. 58, 5471-5484.

Juteau, F., Masotti, V., Bessière, J.M., Dherbomez, M., Viano, J., 2002. Antibacterial and antioxidant activities of Artemisia annua essential oil. Fitoterapia 73, 532-535.

Kallithraka, S., Garcia-Viguera, C., Bridle, P., Bakker, J., 1995. Survey of solvents for the extraction of grape seed phenolics. Phytochem. Anal. 6, 265-267.
Kawaree, R., Okonogi, S., Chowwanapoonpohn, S., Phutdhawong, W., 2008. Chemical composition and antioxidant evaluation of volatile oils from Thai medicinal plants. ISHS Acta Horticult. 786, 209-215.

Kordali, S., Cakir, A., Mavi, A., Kilic, H., Yildirim, A., 2005. Screening of chemical composition and antifungal and antioxidant activities of the essential oils from three Turkish Artemisia species. J. Agric. Food Chem. 53, 1408-1416.

Korekar, G., Stobdan, T., Singh, H., Chaurasia, O.P., Singh, S.B., 2011. Phenolic content and antioxidant capacity of various solvent extracts from seabuckthorn (Hippophae rhamnoides L.) fruit pulp, seeds, leaves and stem bark. Acta Alimentaria 40, 449-458.

Lai, J.-P., Lim, Y.H., Su, J., Shen, H.-M., Ong, C.N., 2007. Identification and characterization of major flavonoids and caffeoylquinic acids in three Compositae plants by LC/DAD-APCI/MS. J. Chromatogr. B 848, 215-225.

Li, G.-X., Liu, Z.-Q., 2009. Unusual antioxidant behavior of $\alpha$ - and $\gamma$-Terpinene in protecting methyl linoleate, DNA, and erythrocyte. J. Agric. Food Chem. 57, 3943-3948.

Lopes-Lutz, D., Alviano, D.S., Alviano, C.S., Kolodziejczyk, P.P., 2008. Screening of chemical composition, antimicrobial and antioxidant activities of Artemisia essential oils. Phytochemical 69, 1732-1738.

M R, V.-r., 2009. Chemical composition and antimicrobial activity of the essential oil of Artemisia annua L. from Iran. Pharmac. Res. 1, 21-24.

Ma, C.-M., Hattori, M., Chen, H.-B., Cai, S.-Q., Daneshtalab, M., 2008. Profiling the phenolic compounds of Artemisia pectinata by HPLC-PAD-MS ${ }^{n}$. Phytochem. Anal. 19, 294-300.

Mabry, T.J., Markham, K.R.,Thomas, M.B., 1970. The ultraviolet spectra of flavones and flavonols, isoflavones, dihydroxyflavonols, The Systematics Identification of Flavonoids, Springer-Verlag, New York, NY.

Magalhães, P.M., Pereira, B., Sartoratoo, A., 2004. Yields of antimalarial Artemisia annua L. species. ISHS Acta Horticult. 629, 421-424.

Magalhães, L.M., Segundo, M.S., Reis, S., Lima, J.L.F.C., 2008. Methodological aspects about in vitro evaluation of antioxidant properties. Anal. Chim. Acta 613, 1-19.

Reale, S., Fasciani, P., Pace, L., Angelis, F.D., Marcozzi, G., 2011. Volatile fingerprints of artemisinin-rich Artemisia annua cultivars by headspace solid-phase microextraction gas chromatography/mass spectrometry. Rapid Commun. Mass Spectrom. 25, 2511-2516.

Shang, Z.H., Hou, Y., Long, R.J., 2012. Chemical composition of essential oil of Artemisia nanschanica Krasch. from Tibetan plateau. Ind. Crops Prod. 40, 35-38.

Verma, R.K., Chauhan, A., Verma, R.S., Gupta, A.K., 2011. Influence of planting date on growth, artemisinin yield, seed and oil yield of Artemisia annua L. under temperate climatic conditions. Ind. Crops Prod. 34, 860-864.

Vukics, V., Ringer, T., Kery, A., Bonn, G.K., Guttman, A., 2008. Analysis of heartsease (Viola tricolor L.) flavonoid glycosides by micro-liquid chromatography coupled to multistage mass spectrometry. J. Chromatogr. A 1206, 11-20.

Yoon, K.D., Chin, Y.-W., Yang, M.H., Kim, J., 2011. Separation of anti-ulcer flavonoids from Artemisia extracts by high-speed countercurrent chromatography. Food Chem. 129, 679-683.

Zheng, W., Wang, S.Y., 2001. Antioxidant activity and phenolic compounds in selected herbs. J. Agric. Food Chem. 49, 5165-5170. 